\title{
Berberine Suppresses Bladder Cancer Cell Proliferation by Inhibiting JAK1-STAT3 Signaling via Upregulation of miR-17-5p
}

\section{Yangyang Xia}

Shandong University Qilu Hospital

\section{Shouzhen Chen}

Shandong University Qilu Hospital

Jianfeng Cui

Shandong University Qilu Hospital

Yong Wang

Shandong University Qilu Hospital

Xiaochen Liu

Shandong University

\section{Yangli Shen}

Shandong University

\section{Li Gong}

Shandong University

\section{Xuewen Jiang}

Shandong University Qilu Hospital

\section{Wenfu Wang}

Shandong University Qilu Hospital

\section{Yaofeng Zhu}

Shandong University Qilu Hospital

\section{Shuna Sun}

The Affiliated Hospital of Shandong University of Traditional Chinese Medicine

Jiaxiang Li

Shandong University

\section{Yongxin Zou}

Shandong University

Benkang Shi ( $\nabla$ bkang68@sdu.edu.cn )

Shandong University Qilu Hospital 
Keywords: bladder cancer, berberine, JAK1, STAT3, miR-17-5p

Posted Date: December 29th, 2020

DOI: https://doi.org/10.21203/rs.3.rs-135605/v1

License: (c) (1) This work is licensed under a Creative Commons Attribution 4.0 International License. Read Full License 


\section{Abstract}

Background: Berberine (BBR), an active component extracted from Coptis chinensis, has shown antitumor effects in multiple tumors. However, the underlying mechanisms haven't been fully elucidated. In this study, the effects of BBR on bladder cancer $(\mathrm{BCa})$ cells and the underlying mechanisms were investigated both in vivo and vitro.

Methods: MTT, colony formation, EdU incorporation assays and xenograft tumor models were performed to evaluate the anti-proliferation effects of BBR on BCa cells in vivo and vitro. The roles of BRR in migration and invasion of $\mathrm{BCa}$ cells were investigated by wound-healing, transwell migration and invasion assays. The apoptosis and senescence induced by BBR were determined by flow cytometry, tunel assay and senescence-associated $\beta$-galactosidase (SA- $\beta$-gal) activity assay. Potential candidate targets were screened using western blot. The relationships between miR-17-5p and JAK1-STAT3 pathway were then predicted using in-silico analysis and identified by dual-luciferase reporter assay. The regulatory mechanism of miR-17-5p mediated suppression of JAK1-STAT3 pathway caused by BBR treatment was validated by qRT-PCR and western blot.

Results: We found that BBR showed significant cytotoxic effects against bladder cancer (BCa) cell lines both in vivo and vitro, with much lower cytotoxic effects on human normal urothelial cell line SV-HUC-1. BBR treatment induced DNA replication defects and cycle cell arrest, resulting in apoptosis or cell senescence, depending on p53 status, in BCa cells. Mechanically, BBR exerted anti-tumor effects on BCa cells through inhibiting JAK1-STAT3 signaling via promoting expression of miR-17-5p, which directly bound to 3' UTR of JAK1 and STAT3 and downregulated their expressions.

Conclusion: Our results demonstrate that BBR exerts anti-tumor effects through perturbing JAK1-STAT3 signaling via upregulating expression of miR-17-5p in BCa cells, and BBR may serve as a potential therapeutic option for BCa treatment.

\section{Background}

Bladder cancer (BCa) is the 10th most common malignancy globally, with an estimated 549,400 new cases and 199,900 cancer-related deaths in $2018^{1}$. The majority of BCa is pathologically classified as urothelial carcinomas ${ }^{2}$. Overall, about $75 \%$ of BC patients initially present with non-muscle invasive bladder cancer (NMIBC), and the primary choice for treatment is transurethral resection of bladder tumor (TURBT) followed by intravesical therapy ${ }^{3}$. Although under intensive management, approximately half of NMIBC cases recur and $10-15 \%$ would progress to muscle-invasive bladder cancer (MIBC) or metastasis within 5 years $^{2}$. For those patients with MIBC, radical cystectomy with lymphadenectomy is still considered as standard treatment. Cisplatin-based chemotherapy is recommended in advanced patients. However, the 5-year survival rate is under $50 \%$ due to chemo-resistance and relapse. In recent years, checkpoint blockade immunotherapy (ICl) and FGFR2/3 target therapy have emerged as potential 
therapeutic strategies for $\mathrm{BC}$ treatment ${ }^{4}$. But these medicines are applicable for certain patients and the objective response rate (ORR) is limited ${ }^{5-7}$. Thus, it is vital to explore novel therapeutic drugs for $\mathrm{BCa}$.

Comprehensive genomic analysis reveals the genomic landscape of $\mathrm{BCa}$ and identifies a number of molecular targets ${ }^{8}$. Increasing evidence shows that signal transducer and activator of transcription 3 (STAT3) plays a vital role in tumorigenesis and development of $\mathrm{BCa}^{9,10}$. Generally, protein ligands in tumor microenvironment activate STAT3 via its phosphokinase-the Janus kinase (JAK), and phosphorylated STAT3 monomer is dimerized to translocate into nucleus and promote transcription of target genes. Phosphorylated STAT3 levels are shown to be upregulated in BCa tissues, and aberrant activation of STAT3 could promote proliferation, metastasis and acquired drug-resistance of $\mathrm{BCa}^{9-11}$. Transgenic mice overexpressing constitutively active STAT3 in bladder epithelium develop invasive bladder cancer ${ }^{9}$. Moreover, blockade of JAK-STAT3 pathway elicits significant anti-tumor effect and reverses resistance in $\mathrm{BCa}$ both in vitro and vivo ${ }^{11-13}$. Therefore, JAK-STAT3 pathway may be a potential target for BCa treatment ${ }^{14}$. Several selective inhibitors targeting STAT3 are undergoing preclinical and clinical researches, however, no one has been approved for clinical cancer treatment due to limited efficacy and unexpected side effects ${ }^{15}$.

Natural products, which are valuable sources in pharmaceutical development, have a reputation for strong potency with minimal side effects. Therefore, the molecular mechanisms underlying the effects of natural products are needed to extensively understand for further pharmaceutical development. Berberine (BBR), derived from Chinese medicinal plant Coptis chinensis, has a proven medical history as an Ayurvedic and Chinese medicine for its therapeutic effects on microbial-related or -independent gastrointestinal disorders ${ }^{16}$. As the most thoroughly studied natural plant alkaloid in the world, BBR has been reported to possess numerous pharmacological activities including antioxidant, anti-inflammatory, organ-protective activities ${ }^{17}$, and recent studies have discovered additional clinical indications. Oral administration of BBR is proved to display beneficial effects in treatment of patients with hypercholesterolemia and diabetics ${ }^{17,18}$. Notably, its antineoplastic effects have been investigated in a broad spectrum of cancer cells. Particularly, BBR has been shown to inhibit proliferation and migration of $\mathrm{BCa}$ cells, and induce DNA damage and apoptosis in BCa cells ${ }^{19,20}$. however, the underlying molecular mechanisms of BBR in attenuating BCa are still poorly understood.

In this study, we investigated the role and underlying molecular mechanisms of BBR in BCa cells. Our results demonstrated that BBR induced apoptosis in p53 deficient while caused senescence in p53 proficient BCa cells via targeting JAK1-STAT3 signaling. We further showed that the inhibitory effect of BBR on JAK1-STAT3 pathway is through upregulating miR-17-5p.

\section{Materials And Methods}

\section{Cell culture and manipulation}


Human BCa cell lines T24, 5637 and immortalized human uroepithelial cell SV-HUC-1 were purchased from American type culture collection. Human BCa cell line 253J was purchased from Cell Bank of the Chinese Academy of Science. All cell lines were identified using short tandem repeat markers. Human BCa cell lines, including T24, 5637 and 253J were cultured in RPMI-1640 medium (\#11875093, Gibco Life Technologies, Carlsbad, CA, USA,). SV-HUC-1 cell line was maintained in F12K medium (\#CM10025, Macgene Technology Ltd, Beijing, China,). All medium was supplemented with $10 \%$ fetal bovine serum (\#10270-106, Gibco), 100U/ml penicillin and $100 \mu \mathrm{g} / \mathrm{ml}$ streptomycin. All cells were maintained at $37^{\circ} \mathrm{C}$ in a humidified atmosphere with $5 \% \mathrm{CO}_{2}$.

Lentiviral vector for overexpressing STAT3 was purchased from Genechem (Shanghai, China). Lentiviral vectors including shRNA targeting p53 (\#19119), overexpression of JAK1 (\#23932) and JAK2 (\#81756) were purchased from Addgene (Cambridge, MA, USA). The miR-17-5p mimics and inhibitor were purchased from GenePharma. Transfections of miR-17-5p mimics/inhibitors and lentiviral transduction were performed as previously described ${ }^{21}$.

\section{Reagents and antibodies}

Berberine chloride (\#B3251), MG132 (\#M8699), and 3-(4, 5-dimethylthiazol-2-yl)-2,5-diphenyltetrazolium bromide (MTT, \#M2128) were purchased from Sigma (St. Louis, MO, USA). Stattic (\#HY-13818) was purchased from MedChemExpress (Monmouth Junction, NJ, USA). Berberine chloride and stattic were dissolved in Dimethyl sulfoxide (DMSO), and MTT was dissolved in phosphate-buffered solution (PBS). Primary antibodies for western blot were presented in Supplementary Table 1.

\section{MTT, colony formation, and EdU incorporation assays}

Cell proliferation and viability were measured by MTT, colony formation and EdU incorporation assays, which were performed as previously described ${ }^{22}$.

\section{Wound-healing, transwell migration, and invasion assays}

To detect the migration and invasion capacities of BCa cells, the wound-healing, transwell migration, and invasion assays were performed as previously described ${ }^{21}$.

\section{Senescence-associated $\beta$-galactosidase (SA- $\beta$-gal) activity and tunel assay}

Senescence-associated $\beta$-galactosidase (SA- $\beta$-gal) activity was detected using the Senescence $\beta$ Galactosidase Staining Kit (\#C0602, Beyotime Biotechnology, Jiangsu, China) and performed as previously described ${ }^{22}$.

Tunel assay was carried out using one-step Tunel Apoptosis Assay Kit (\#C1089, Beyotime) and performed as previously described ${ }^{22}$. 
Intracellular ROS levels were measured with Reactive Oxygen Species Assay Kit (\#S0033S, Beyotime). DNA damages were measured with alkaline comet assay using Single Cell Gel Electrophoresis Assay Kit (\#4251-050-K, Trevigen, Gaithersburg, MD, USA). ROS assay and alkaline comet assay were performed as previously described ${ }^{23}$.

\section{Apoptosis assay}

Percentage of apoptotic cells was evaluated by Annexin V-FITC apoptosis Kit (\#556547, BD Biosciences, Shanghai, China) according to the manufacturer's protocols. The apoptosis assay was performed as previously described ${ }^{23}$.

\section{Protein extraction and western blot}

Total proteins were extracted using RIPA lysis buffer containing proteinase and phosphatase inhibitors. Nuclear and cytoplasmic proteins were isolated with Nuclear and Cytoplasmic Protein Extraction Kit (\#P0027, Beyotime) according to the manufacturer's instructions. Western blot analysis was performed as described previously ${ }^{21}$. The primary antibodies are listed in Supplementary Table 1.

\section{RNA extraction and real-time quantitative PCR (qRT-PCR) assay}

Isolation of total RNA and qRT-PCR assays were performed as described previously ${ }^{21}$. Primer sequences used for qRT-PCR were listed in Supplementary Table 2. All primers used for miRNA qRT-PCR were purchased from GeneCopoeia (Rockville, MD, USA).

\section{Luciferase assay}

The segments of JAK1 and STAT3 3'UTR containing wild-type and mutated miR-17-5p binding site were cloned into the pmir-GLO vectors (\#E1330, Promega) which were purchased from BioSune (Shanghai, China). The luciferase assays were performed as previously described ${ }^{21}$.

\section{In vivo tumor xenografts}

For generation of $\mathrm{BCa}$ xenografts, $5 \times 10^{6} \mathrm{~T} 24$ cells suspended in $100 \mu \mathrm{l}$ PBS were subcutaneously injected into the dorsal flank of female 5- to 6-week-old BALB/c nude mice (Vital River Laboratory Animal Technology Co. Ltd, Beijing, China). One week later, the tumor-bearing mice were randomly divided into 2 groups and treated daily by oral gavage with DMSO and BBR $(200 \mathrm{mg} / \mathrm{kg}$ body weight) respectively for two weeks. All mice were euthanized after 2 weeks and tumors were harvested. Tumors were measured using calipers, and tumor volume was calculated by the formula: Tumor volume $\left(\mathrm{mm}^{3}\right)=0.5 \times$ tumor length $(\mathrm{mm}) \times$ [tumor width $\left.(\mathrm{mm})^{2}\right]$. After weighing, tumor tissues were snap-frozen or embedded in paraffin for further analysis. The experimental protocols were approved by the Institutional Animal Care and Use Committee of Shandong University and all procedures were performed in compliance with the institutional guidelines. 


\section{Immunohistochemistry analysis}

The tumor tissues were collected, fixed with formalin buffer, and embedded in paraffin. Immunohistochemical (IHC) staining was performed as previously described ${ }^{21}$.

\section{Statistical analysis}

All results were expressed as mean $\pm S D$. The statistical analysis and box plots were carried out using GraphPad Prism 6 software (La Jolla, CA, USA). The statistical comparisons were calculated by unpaired two-tailed Student's $t$-test and were considered significant if $p<0.05$. And levels of significance were presented at ${ }^{*} P<0.05,{ }^{*} P<0.01$, and ${ }^{* * *} P<0.001$ respectively.

\section{Results}

\section{BBR inhibits proliferation and induces cell cycle arrest in BCa cells.}

We first evaluated the anti-proliferation effects of BBR on BCa cells. MTT assays showed that BBR significantly decreased cell viability in a dose-dependent manner (Fig. 1a and Supplementary Fig. 1a). The results of colony formation assay also showed that BBR induced a concentration-dependent decrease in the number of cell colonies (Fig. 1b). Moreover, BCa cells treated with BBR exhibited much smaller colonies (Supplementary Fig. 1b). Next, EdU incorporation assays were utilized to detect the effects of BBR on DNA replication. Treatment of BBR significantly decreased the percentage of EdU positive cells in BCa cells (Fig. 1c and Supplementary Fig. 1c-e). Notably, the inhibitive effect of BBR on cell viability, colony formation and DNA replication were much less evident in the human urothelial cells (SV-HUC-1) (Fig. 1a-C and Supplementary Fig. $1 \mathrm{a}, 1 \mathrm{f}$ and $1 \mathrm{~g}$ ), suggesting that the normal urothelial cells were less sensitive to BBR treatment than that of BCa cells.

Next, we investigated the underlying mechanisms of anti-proliferative effects of BBR in BCa cells. Cell cycle distribution analysis by flow cytometry showed that the effects of BBR on cell cycle varied in $\mathrm{BCa}$ cell lines. BBR treatment increased the proportion of cells in G0/G1 phase, accompanied by a decrease in $S$ phase and G2/M in T24 cells (Fig. 1d). While, BBR elevated proportion of S phase in 5637 cells, along with a decline in proportion of G0/G1 and G2/M phase (Fig. 1d). Treatment of 253J cells with BBR resulted in an increase in proportion of G2/M phase (Fig. 1d). Western blots analysis on cell cycleassociated proteins revealed that the protein levels of CDK1, CDK2, CDK4, cyclin A, cyclin D1 and cyclin E were significantly decreased in BBR-treated T24, 5637 and 253J cells (Fig. 1e and Supplementary Fig. 1h).

We then established a xenograft model to further assess the effects of BBR on tumor growth in vivo. Applications of BBR significantly suppressed tumor growth of T24 cells (Fig. 1f). Consistently, the tumor volume and tumor weight were obviously reduced in BBR-treatment group (Fig. 1g). Moreover, immunohistochemistry (IHC) assays showed that BBR administration significantly decreased Ki-67 
expression levels (Fig. 1h). Taken together, these results suggest that BBR inhibits the proliferation of BCa cells both in vivo and vitro.

\section{Bbr Suppresses Migration And Invasion Of Bca Cells}

We further determined the roles of BRR in migration and invasion of BCa cells. Wound healing assays showed that BCa cells treated with BBR exhibited a lower wound closure rate compared with that of cells treated with DMSO (Fig. 2a and Supplementary Fig. 2a). Transwell assays were further verified the decreased capabilities of migration in BBR-treated BCa cells (Fig. 2b and Supplementary Fig. 2b). Matrigel invasion assays also showed BBR treatment resulted in a smaller number of invasive cells (Fig. 2c-e). These results indicated that BBR significantly attenuated the abilities of migration and invasion of BCa cells. Considering the vital role of Epithelial-Mesenchymal Transition (EMT) in regulating cancer cell migration and invasion, we next detected expression levels of several marker proteins involved in EMT. Western blot analysis revealed that BBR significantly down-regulated the expression of Snail, NCadherin and Vimentin in BCa cells (Fig. 2f-h), suggesting that BBR suppresses EMT in BCa cells. Besides, BBR treatment suppressed mRNA expression levels of MMP2 and MMP9 (Fig. 2i), two important genes for tumor metastasis.

\section{BBR induces apoptosis or senescence in BCa cells in a p53-dependent way}

Previous studies reported that BBR exerted anti-proliferative effects through apoptosis induction in various cancer cells. Thus, we next investigated whether BBR caused apoptosis in BCa cells. As shown in Fig. 3a-c, treatment of BBR significantly increased the percentage of apoptotic cells in T24 and 5637 cells, however, no significant changes in apoptotic cells percentage were observed in 253J cells. Consistently, the percentages of Tunel positive cells were significantly increased in BBR-treated T24 and 5637 cells (Fig. $3 \mathrm{~d}$ and Supplementary Fig. 3a-b), but not in $253 \mathrm{~J}$ cells (data not shown). Moreover, the levels of cleaved caspase-3, a marker of apoptosis, were up-regulated in T24 and 5637 cells with BBR treatment (Fig. 3e). In contrast, BBR treatment didn't affect the levels of cleaved caspase-3 in 253J cells (Supplementary Fig. 3c).

BBR was reported to induce cell apoptosis through accumulation of reactive oxygen species (ROS) and oxidative DNA damages. The comet assays showed that the mean olive tail moment values were significantly increased in T24 and 5637 cells treated with BBR (Fig. 3f-g). Moreover, the intracellular ROS levels were also drastically increased with increasing concentrations of BBR in these two cell lines (Fig. 3h), suggesting BBR caused ROS accumulation and DNA damage in T24 and 5637 cells. In contrast, BBR-treated 253J cells displayed similar olive tail moment values and ROS levels with that of DMSOtreated cells respectively (Supplementary Fig. 3d-e), indicating that the underlying mechanisms about proliferative inhibition of BBR might be different between 253J and the other two BCa cell lines. To investigate the mechanisms of BBR in cell proliferation regulation in 253J cells, we next performed SA- $\beta$ gal activity assays to evaluate the effect of BBR on cell senescence. The results showed that BBR 
treatment significantly increased the number of SA- $\beta$-gal positive cells (Fig. 3i), substantiating that BBR induces cell senescence of $253 \mathrm{~J}$ cells.

p53 is considered as one of the most critical tumor suppressors, and previous studies showed that cancer cells harboring wild-type or mutant-type p53 displayed different outcomes of drug-induced cell death ${ }^{24}$. The results that BBR induced apoptosis in p53 mutant T24 and 5637 cells, while induced cell senescence in p53 wild-type 253J cells prompted us to investigate whether the apoptosis induced by BBR in BCa cells was dependent on p53 status. 253 J cell line with stable p53 knock-out (253J p53-KO) was established (Fig. 3j-k). As shown in Fig. 3l, BBR treatment in 253J p53-KO cells caused a significant increase in apoptotic cells percentage compared with that of negative control (253J NC) cells. Besides, knockdown of p53 significantly attenuated the cell senescence caused by BBR in $253 \mathrm{~J}$ cells (Fig. $3 \mathrm{~m}$ ), suggesting that the cell fate decisions, by BBR, depend on p53 function in BCa cells.

\section{BBR down-regulates the expression and activity of STAT3 in BCa cells.}

To further investigate the mechanism that BBR inhibits proliferation of BCa cells, the levels of phosphorylated ERK (p-ERK), phosphorylated AKT (p-AKT) and phosphorylated STAT3 (p-STAT3), which were known as important regulators in cancer progression, were examined. As shown in Fig. 4a, no significant changes in protein levels of p-ERK and p-AKT were observed in BBR treated T24 and 5637 cells. In contrast, both the p-STAT3 and total STAT3 protein levels were significantly decreased in BBR treated T24 and 5637 cells (Fig. 4b). Given that nuclear translocation of activated STAT3 was necessary for transcription of its target genes, the levels of cytoplasmic and nuclear STAT3 and p-STAT3 were detected. As shown in Fig. 4c, both cytoplasmic and nuclear STAT3 and p-STAT3 protein levels were down-regulated by BBR. Furthermore, luciferase analysis confirmed an obvious decrease in transcriptional activity of STAT3 in BBR-treated cells (Fig. 4d). Together, these results suggest that the activity of STAT3 was suppressed by BBR in BCa cells.

We next sought to evaluate whether STAT3 inhibition was responsible for BBR-mediated proliferation suppression in BCa cells. Stattic, a STAT3 inhibitor, significantly inhibited cell viability in a concentrationdependent manner (Fig. 4e). Importantly, similar with that of BBR, stattic treatment induced apoptosis in T24 cells, while induced cell senescence in 253J cells (Fig. 4f-4 g and Supplementary Fig. 4a-b). Moreover, stattic treatment in 253J p53-KO cells also significantly induced apoptosis accompanied by reduction of cell senescence (Fig. $4 \mathrm{~h}-4 \mathrm{i}$ ), resembling that of BBR treated cells. Altogether, these results suggest that the proliferation inhibition, apoptosis and senescence induction caused by BBR in BCa cells are potentially dependent on downregulation of STAT3.

\section{Bbr Inhibits Stat3 Activation Through Suppressing Jak1}

To further investigate whether the anti-tumor effect of BBR on BCa cells was mediated by STAT3 expression inhibition, we established STAT3 overexpressed T24 and 5637 cells (STAT3 ${ }^{\text {OVE }}$ ) (Fig. 5a). Western blot analysis showed that overexpression of exogenous STAT3 significantly restored the reduced 
endogenous total STAT3 protein level caused by BBR (Fig. 5b). Of note, BBR also significantly decreased the exogenous STAT3 expression (Fig. 5b). MTT assays showed that expression of exogenous wild-type STAT3 didn't rescue BCa cells from proliferative inhibition caused by BBR (Fig. 5c). EdU incorporation assays showed that the number of EdU positive cells was statistically increased in STAT ${ }^{\text {OVE }}$ cells than that of control cells under BBR treatment, however, it still significantly less than that of DMSO treated cells (Fig. 5d and Supplementary Fig. 5a), indicating the protective effect of introduction of exogenous STAT3 on DNA replication is not significantly effective to attenuate the efficacy of BBR in BCa cells. Consistently, overexpression of STAT3 didn't obviously restore p-STAT3 levels downregulated by BBR (Fig. 5e). Together, these results indicate that BBR-induced proliferation and STAT3 activity inhibition are not only mediated by decreasing STAT3 expression.

Given the critical role of Janus kinases (JAKs) in activating STAT3, we next examined the expression levels of JAK1 and JAK2. As shown in Fig. $5 f$ and Supplementary Fig. 5b, BBR significantly decreased both the total and phosphorylated protein levels of JAK1 and JAK2 in T24 and 5637 cells. Importantly, overexpression of JAK1, but not JAK2, significantly restored p-STAT3 levels downregulated by BBR (Fig. 5g-i and Supplementary Fig. 5c), indicating that JAK1 is a key target through which BBR exerted antitumor effects on BCa cells. As expected, overexpression of JAK1 significantly attenuated the BBRinduced proliferation inhibition, cell cycle arrest and apoptosis (Fig. 5j-m and Supplementary Fig. 5d). Together, these results suggested that, BBR suppresses JAK1 expression to inhibit STAT3 activation in BCa cells.

\section{Bbr Suppresses Jak1-stat3 Signaling Via Upregulation Of Mir-17-5p}

To elucidate the mechanisms that BBR downregulates JAK1 and STAT3 expression, we first detected their mRNA levels. As shown in Fig. 6a and Supplementary Fig. 6a, BBR treatment did not decrease mRNA levels of JAK1 and STAT3 in 5637 and T24 cells. Moreover, the decreased protein levels of JAK1 and STAT3 caused by BBR treatment could not be restored by MG132 (Supplementary Fig. 6b). All these results suggest that downregulation of JAK1 and STAT3 expression induced by BBR was not mediated by regulating transcription or protein degradation.

MicroRNAs (miRNAs) degrades or inhibits translation of downstream target genes via binding to complementary mRNAs. Accumulating evidence has proved that dysregulated miRNAs induced by drug treatment were important regulators of cell fate in cancer progression ${ }^{25}$. Thus, we then investigated whether BBR downregulated JAK1 and STAT3 via miRNAs. The online softwares- TargetScan and miR WALK were utilized to predict miRNAs targeting both JAK 1 and STAT3, and 5 candidate miRNAs were identified (Fig. 6b). qRT-PCR assays showed that only miR-17-5p level was upregulated by BBR treatment (Fig. 6c). Further analysis revealed that BBR promoted expression of miR-17-5p in a concentrationdependent manner (Fig. 6d). Importantly, transfection of miR-17-5p mimics significantly decreased protein levels of JAK1 and STAT3 in T24 and 5637 cells, leading to downregulate p-STAT3 expression 
(Fig. 6e). Moreover, miR-17-5p mimics significantly inhibited cell proliferation (Fig. 6f) in 5637 cells, similar with that of BBR. To determine whether miR-17-5p directly binds to $3^{\prime}$ UTR regions of JAK1 and STAT3 as in-silico prediction (Supplementary Fig. 6c), dual-reporter luciferase assays were performed. As shown in Fig. $6 \mathrm{~g}$, the activities of reporters containing wild-type JAK1 and STAT3 3'UTR, but not the miR17-5p binding sites mutant $3^{\prime} U T R$, were decreased by transfection of miR-17-5p mimics. Collectively, these data indicate that miR-17-5p directly binds to 3' UTR of JAK1 and STAT3 and inhibits their expression in BCa cells.

We then investigated whether BBR downregulated JAK1 and STAT3 expression via miR-17-5p regulation. As shown in Fig. 6h, transfection with miR-17-5p inhibitors partially blocked the decreased expressions of JAK1, p-STAT3 and STAT3 caused by BBR treatment. Furthermore, luciferase reporter assays showed that the wild-type JAK1 and STAT3 3'UTR activities were significantly decreased by BBR. However, these inhibitory effects were abolished by mutating the miR-17-5p binding sites (Fig. 6i), suggesting that BBR perturbed JAK1-STAT3 pathway via upregulation of miR-17-5p.

\section{The effect of BBR on miR-17-5p-JAK1-STAT3 pathway in vivo.}

Last, we examined JAK1 and STAT3 expression levels in xenograft tumors. The results showed that mRNA levels of JAK1 and STAT3 were not significantly altered by BBR treatment (Fig. 7a-b), however, miR-17-5p expression was obviously upregulated in BBR-treatment tumors (Fig. 7c). Moreover, the results of western blot and IHC analyses on xenograft tumors confirmed that BBR decreased JAK1, STAT3 and pSTAT3 protein levels in vivo (Fig. 7d-e). Collectively, these results suggest that BBR promotes miR-17-5p expression to suppress JAK1 and STAT3 both in vivo and vitro.

\section{Discussion}

Despite great progresses in treatment of $\mathrm{BCa}$, emerging medicines only provide therapeutic benefits in certain applicable patients. Most patients ultimately undergo cancer progression, which is still a great challenge for $\mathrm{BCa}$ clinical management ${ }^{4}$. Therefore, novel drugs for $\mathrm{BCa}$ treatment are highly needed. Previous studies have reported that BBR inhibits proliferation, migration and induces apoptosis in T24 cells ${ }^{19,20}$. In this study, we demonstrated that BBR significantly inhibited proliferation, migration and invasion of BCa cells. Notably, BBR exhibited much less evident anti-proliferation effects on human normal urothelial cells (SV-HUC-1), suggesting the potential of BBR in BCa treatment. Moreover, we found that BBR induced apoptosis in p53 deficient while caused senescence in p53 proficient BCa cells. Further analyses on the underlying mechanism revealed that BBR inhibited JAK1-STAT3 signaling pathway to exert its cytotoxic effects.

Hyperactivation of STAT3 occurs in over 70\% of human malignant cancers, including bladder cancer, which usually results in poor patient outcomes ${ }^{26}$. Previous studies have reported the important role of STAT3 in BCa. Recruitment and activation of STAT3 induce epithelial-mesenchymal transition (EMT) to maintain metastatic potential of $\mathrm{BCa}^{10,27}$. STAT3 activation in urothelial stem cells leads to development 
of invasive $\mathrm{BCa}^{9}$, and activated STAT3 triggers self-renewal of bladder cancer stem cells, which plays a crucial role in tumor initiation, progression and drug resistance ${ }^{11,28}$. On the contrary, blocking STAT3 signaling impairs progressions of bladder cancer both in vitro and vivo ${ }^{13,29}$. Moreover, STAT3 inhibition sensitizes bladder cancer to anti-PD-L1 immune therapy and reverses cisplatin resistance ${ }^{11-13}$, suggesting that STAT3 may serve as an attractive target for BCa management. BBR was reported to inhibit STAT3 activation in several malignant tumors. BBR suppresses tumorigenesis and tumor growth via inhibiting STAT3 activation induced by cancer-associated fibroblasts (CAFs) in colorectal cancer and nasopharyngeal carcinoma ${ }^{30,31}$. Activation of STAT3 mediated by COX2-PGE2 pathway could be inhibited by BBR, which leads to inhibition of migration and invasion in colorectal cancer ${ }^{32}$. Besides, BBR is proved to enhance the antitumor effects of EGFR inhibitors in gastric cancer by inhibiting STAT3 activation $^{33}$. Inhibition of STAT3 activation by BBR is also reported in autoimmune myocarditis, ulcerative colitis, ectopic stromal cells, podocytes and ocular Behcet's disease via down-regulating expression of IL6, CXCL12, IL-17, etc ${ }^{34-38}$. All these results indicate that STAT3 might be a key target through which BBR exerts its effects. Notably, the majority of these studies showed that though activation of STAT3 was decreased by BBR treatment, total STAT3 protein levels were not affected. Here, we found that BBR downregulated expression of both total and phosphorylated STAT3 in BCa cells. Selectively blocking STAT3 inhibited cell viability and induced apoptosis or senescence in BCa cells, similar to that of BBR. While, overexpression of exogenous STAT3 didn't attenuate the effects of BBR or restore p-STAT3 levels downregulated by $\mathrm{BBR}$ in $\mathrm{BCa}$ cells, indicating that BBR exerted cytotoxic effects in BCa cells via suppressing both the total expression and activation of STAT3.

In tumor microenvironment, activation of STAT3 in response to cytokines is mediated by JAKs ${ }^{39}$. Suppression of JAKs results in abolishing STAT3 activation, which leads to repress progression and reverse chemoresistance in multiple cancers, including BCa. Importantly, several clinical studies have proved JAK inhibitors as an attractive candidate target for various malignancies ${ }^{40}$. BBR is reported to inhibit expression and phosphorylation of JAK1 and induces apoptosis in keratinocytes ${ }^{23}$, however, the underlying mechanism is poorly understood. Here we demonstrated that BBR inhibited expression of JAK1, and overexpression of JAK1 effectively restored STAT3 activation down-regulated by BBR, and attenuated BBR-induced proliferation inhibition, cell cycle arrest and apoptosis, indicating that JAK1 is the key target of BBR in BCa cells. Until now, a large number of studies have demonstrated the effect of BBR on repressing STAT3 activation through multiple ways, however, seldom research has discovered inhibition of JAK1-STAT3 signaling via suppressing both expression and activation of STAT3. In this study, we showed that BBR down-regulated expressions of both JAK1 and STAT3, which resulted in down-regulation of STAT3 activity. To our knowledge, it is the first time that BBR is proved to inhibit both JAK1 and STAT3 total protein expression in neoplasm. Our results revealed further regulatory mechanism of BBR on JAK1-STAT3 axis, which would provide supporting evidence for potential clinical application.

MicroRNAs (miRNAs) are endogenously non-coding RNAs about 19-23 nucleotides in length. miRNAs bind with imperfect complementarity to mRNAs of their target genes and facilitate translational repression or mRNA degradation. Accumulating evidence suggests that aberrant expressions of miRNAs 
have important roles in the initiation, development and metastasis of bladder cancer ${ }^{41}$. Multiple miRNAs are shown to directly bind to $3^{\prime}$ UTR of JAK1 and STAT3 respectively in tumors ${ }^{42}$. Here, we founded that BBR treatment promoted expression of miR-17-5p in BCa cells. Overexpression of miR-17-5p directly suppressed expressions of JAK1 and STAT3, which resulted in proliferative inhibition. Importantly, transfection of miR-17-5p inhibitors significantly restored JAK1 and STAT3 expression downregulated by $B B R$, suggesting upregulation of miR-17-5p is a key step through which BBR exerts its effects on repressing JAK1 and STAT3 expression.

miR-17-5p, a member of miR-17-92 cluster, is involved in a wide range of biological processes. It is reported that miR-17-5p is highly expressed in embryonic cells and its absence in mouse models results in lung hypoplasia, ventricular septal defect and skeletal abnormalities ${ }^{43,44}$. Besides, previously studies also indicate a vital role of miR-17-5p in maturation of immune system and differentiation of immune cells $s^{44,45}$. Accumulating evidence demonstrates the important role of miR-17-5p in malignant tumors. It is reported that miR-17-5p promotes tumor proliferation via targeting PTEN and $p 21^{46}$. Moreover, miR-17-5p directly regulates expressions of TIMP3 and TP53INP1, and functions as a vital oncogenic factor in promoting progression in prostate and gastric cancer ${ }^{47,48}$. However, the role of miR-17-5p in malignant tumors remains ambiguous. It is reported that miR-17-5p suppresses cell growth and promotes apoptosis of cervical cancer cells by also targeting TP53INP1 ${ }^{49}$. The migration and invasion capacities of malignant pleural mesothelioma and hepatocellular carcinoma are suppressed by miR-17-5p via directly targeting KCa1.1 and ERBB3 ${ }^{50,51}$. Besides, systematic analysis identifies miR-17-5p as a metastatic suppressor and miR-17-5p exerts its suppressive effect by targeting ETV1 and AIB1 in breast cancer ${ }^{52,53}$. Notably, miR-17-5p is reported to modulate expressions of STAT3 and CXCL14 ${ }^{48,54}$, another activator of STAT3, indicating that STAT3 might be a vital target through which miR-17-5p functions as tumor suppressor. These results suggest that the role of miR-17-5p in regulating tumor progression is tumor or tissue-specific. Currently, a large number of studies have suggested JAK-STAT3 signaling is an important mediator through which miR-17-5p exerts its effects on multiple processes. Inhibition of miR-17-5p upregulates STAT3 expression and facilitate its nuclear translocation, which subsequently promotes fibroblasts proliferation and migration ${ }^{55}$. Overexpression of STAT3 effectively reverses the effect of miR$17-5 p$ on inducing apoptosis in immortalized mouse podocytes and cardiomyocytes ${ }^{56,57}$. And inhibition of miR-17-5p promotes neurite growth via activating STAT3 ${ }^{58}$. Notably, miR-17-5p is reported to directly target both JAK1 and STAT3 in rheumatoid arthritis fibroblast-like synoviocytes to reduce inflammation ${ }^{59}$. All these results suggest that JAK1-STAT3 signaling is an essential mediator for miR-17-5p. Until now, very few studies have investigated the function of miR-17-5p in BCa. Given that hyperactivation of JAK1/STAT3 signaling is essential for BCa progression, and blocking JAK1 or STAT3 shows obvious antitumor effects in $\mathrm{BCa}^{11,12,26}$, the miR-17-5p-JAK1-STAT3 axis could be envisaged as a potential target in BCa therapy.

\section{Conclusions}


In summary, we demonstrated that BBR effectively inhibited proliferation, migration, invasion and induced apoptosis or senescence of BCa cells. Moreover, we showed that BBR exerted its anti-tumor effects via suppressing JAK1-STAT3 signaling pathway. BBR could promote expression of miR-17-5p and consequently down-regulates JAK1 and STAT3 expression, indicating miR-17-5p-JAK1-STAT3 axis is involved in the anti-tumor activity of BBR in BCa (Fig. 7f). As most available data has shown that BBR was relatively safe in the majority of laboratory and clinical situations ${ }^{60}$, our results provided supporting evidence for the clinical use of BBR in BCa treatment.

\section{Abbreviations}

BCa: bladder cancer; BBR: berberine; NMIBC: non-muscle invasive bladder cancer; MIBC: muscle invasive bladder cancer; TURBT\transurethral resection of bladder tumor; ICl: checkpoint blockade immunotherapy; FGFR2/3: fibroblast growth factor receptor 2/3; ORR: objective response rate; STAT3: signal transducer and activator of transcription 3; JAK1: Janus kinase 1; p53: tumor protein p53; JAK2: Janus kinase 2; MTT: 3-(4, 5-dimethylthiazol-2-yl)-2,5-diphenyltetrazolium bromide; DMSO: Dimethyl sulfoxide; PBS: phosphate-buffered solution; EdU: 5-ethynyl-2'-deoxyuridine; MG132; Z-Leu-leu-leu-al; SA$\beta$-gal: senescence-associated $\beta$-galactosidase; ROS: reactive oxygen species; qRT-PCR: real-time quantitative PCR; Tunel: terminal deoxynucleotidyl transferase mediated dUTP nick-end labeling; RIPA: radio immunoprecipitation assay; UTR: untranslated regions; IHC: Immunohistochemical stain. CDK1: cyclin dependent kinase 1; CDK2: cyclin dependent kinase 2; CDK4: cyclin dependent kinase 4; EMT: epithelial-mesenchymal transition; MMP2: matrix metallopeptidase 2; MMP9: matrix metallopeptidase 9; ERK: extracellular signal-regulated kinase; AKT: serine/threonine kinase 1; OVE: overexpression; NC: negative control; PD-L1: programmed death ligand 1; CAFs: cancer-associated fibroblasts; COX2: cyclooxygenase 2; PGE2: prostaglandin E2; EGFR: epidermal growth factor receptor; IL-6: Interleukin-6; IL17: Interleukin-17; CXCL12: C-X-C motif chemokine ligand 12; PTEN: phosphatase and tensin homolog; TIMP3: TIMP metallopeptidase inhibitor 3; TP53INP1: tumor protein p53 inducible nuclear protein 1; KCa1.1: potassium calcium-activated channel subfamily $M$ alpha 1; ERBB3: erb-b2 receptor tyrosine kinase 3; ETV1: ETS variant transcription factor 1; AIB1: nuclear receptor coactivator amplified in breast cancer 1; CXCL14: C-X-C motif chemokine ligand 14;

\section{Declarations}

\section{Acknowledgement}

We would like to thank Dr. Changshun Shao (State Key Laboratory of Radiation Medicine and Protection, Institutes for Translational Medicine, Soochow University) for kindly providing lentiviral vector for shRNA targeting p53.

\section{Author contributions}


YZo, BS and YX contributed to the conception and design of the study. YX, SC, JC, JL and YW performed the experiments. YS, LG, XJ, WW, YZh and SS performed the data analyses and animal experiment guide. $Y Z o, B S$ and $Y X$ wrote the manuscript. All authors reviewed the manuscript and approved the final versions.

\section{Funding}

This work was supported by the National Natural Science Foundation of China (grants 32070712 and 31671427 to YZo, grant 81804104 to SS, grant 81800672 to SCh, grants 81470987 and 81670687 to BS), the Tai Shan Scholar Foundation to BS, the Science and Technology Development Project of Jinan (grant 201602155 to BS), Natural Science Foundation of Shandong Province (grant ZR2014HQ062 to YZh), Primary Research and Development Plan of Shandong Province (grant 2016GSF201036 to YZh, grant 2019GSF108123 to SCh), and The Fundamental Research Funds of Shandong University (grant $2017 \mathrm{GC008}$ to JL).

\section{Availability of data and materials}

All data generated or analyzed in this study are included in this manuscript and additional files.

\section{Ethics approval and consent to participate}

All animal experimental protocols were approved by the Institutional Animal Care and Use Committee of Shandong University and all procedures were performed in compliance with the institutional guidelines.

\section{Consent for publication}

All authors read and approved the final manuscript.

\section{Conflict of interests}

The authors declare that they have no competing interests

\section{Author details}

${ }^{1}$ The Key Laboratory of Experimental Teratology of Ministry of Education, Department of Medical Genetics, School of Basic Medical Sciences, Cheeloo College of Medicine, Shandong University, Jinan, 250012, China,

${ }^{2}$ Key Laboratory of Urinary Precision Diagnosis and Treatment, Department of Urology, Qilu Hospital, Cheeloo College of Medicine, Shandong University, Jinan, 250012, China,

${ }^{3}$ Department of Dermatology, The Affiliated Hospital of Shandong University of Traditional Chinese Medicine, Shandong Provincial Hospital of Traditional Chinese Medicine, Jinan, Shandong, 250011, China 


\section{References}

1. Bray F, Ferlay J, Soerjomataram I, Siegel RL, Torre LA, Jemal A. Global cancer statistics 2018: GLOBOCAN estimates of incidence and mortality worldwide for 36 cancers in 185 countries. CA Cancer J Clin. 2018;68(6):394-424. doi:10.3322/caac.21492.

2. Knowles MA, Hurst CD. Molecular biology of bladder cancer: New insights into pathogenesis and clinical diversity. Nat Rev Cancer. 2015;15(1):25-41. doi:10.1038/nrc3817.

3. Babjuk M, Burger M, Compérat EM, et al. European Association of Urology Guidelines on Non-muscleinvasive Bladder Cancer (TaT1 and Carcinoma In Situ) - 2019 Update. Eur Urol. 2019;76(5):639-57. doi:10.1016/j.eururo.2019.08.016.

4. Witjes JA, Babjuk M, Bellmunt J, et al. EAU-ESMO Consensus Statements on the Management of Advanced and Variant Bladder Cancer-An International Collaborative Multistakeholder Effort: Under the Auspices of the EAU-ESMO Guidelines Committees. Eur Urol. 2020;77(2):223-50. doi:10.1016/j.eururo.2019.09.035.

5. Gómez de Liaño Lista A, van Dijk N, de V Oria de Rueda G, et al. Clinical outcome after progressing to frontline and second-line Anti-PD-1/PD-L1 in advanced urothelial cancer. Eur Urol. 2020;77(2):269276. doi:10.1016/j.eururo.2019.10.004.

6. Joseph RW, Loriot Y, Perez-Gracia JL, et al. Clinical Characteristics Associated With Early Progression or Long-Term Response From the Phase li Imvigor210 Study: Atezolizumab in Locally Advanced or Metastatic Urothelial Carcinoma. J Urol. 2018;199(4S):e1039. doi:10.1016/j.juro.2018.02.2555.

7. Loriot Y, Necchi A, Park SH, et al. Erdafitinib in Locally Advanced or Metastatic Urothelial Carcinoma. N Engl J Med. 2019;381(4):338-48. doi:10.1056/NEJMoa1817323.

8. Tan TZ, Rouanne M, Tan KT, Huang RYJ, Thiery JP. Molecular Subtypes of Urothelial Bladder Cancer: Results from a Meta-cohort Analysis of 2411 Tumors. Eur Urol. 2019;75(3):423-32. doi:10.1016/j.eururo.2018.08.027.

9. Ho PL, Lay EJ, Jian W, Parra D, Chan KS. Stat3 activation in urothelial stem cells leads to direct progression to invasive bladder cancer. Cancer Res. 2012;72(13):3135-42. doi:10.1158/00085472.CAN-11-3195.

10. Chen Z, Chen X, Xie R, et al. DANCR Promotes Metastasis and Proliferation in Bladder Cancer Cells by Enhancing IL-11-STAT3 Signaling and CCND1 Expression. Mol Ther. 2019;27(2):326-41. doi:10.1016/j.ymthe.2018.12.015.

11. Ojha R, Singh SK, Bhattacharyya S. JAK-mediated autophagy regulates stemness and cell survival in cisplatin resistant bladder cancer cells. Biochim Biophys Acta - Gen Subj. 2016;1860(11, Part A):2484-2497. doi:10.1016/j.bbagen.2016.07.021.

12. Wang WJ, Li CF, Chu YY, et al. Inhibition of the EGFR/STAT3/CEBPD Axis reverses cisplatin crossresistance with paclitaxel in the urothelial carcinoma of the urinary bladder. Clin Cancer Res. 2017;23(2):503-13. doi:10.1158/1078-0432.CCR-15-1169. 
13. Korac-Prlic J, Degoricija M, Vilović K, et al. Targeting Stat3 signaling impairs the progression of bladder cancer in a mouse model. Cancer Lett. 2020;490:89-99. doi:10.1016/j.canlet.2020.06.018.

14. van Kessel KEM, Zuiverloon TCM, Alberts AR, Boormans JL, Zwarthoff EC. Targeted therapies in bladder cancer: an overview of in vivo research. Nat Rev Urol. 2015;12(12):681-94. doi:10.1038/nrurol.2015.231.

15. Gu Y, Mohammad IS, Liu Z. Overview of the STAT-3 signaling pathway in cancer and the development of specific inhibitors. Oncol Lett. 2020;19(4):2585-94. doi:10.3892/ol.2020.11394.

16. Birdsall TC. Berberine. Therapeutic potential of an alkaloid found in several medicinal plants. Altern Med Rev. 1997;2(2):94-103.

17. Kong W-J, Vernieri C, Foiani M, Jiang J-D. Berberine in the treatment of metabolism-related chronic diseases: A drug cloud (dCloud) effect to target multifactorial disorders. Pharmacol Ther. 2020;209:107496. doi:10.1016/j.pharmthera.2020.107496.

18. Feng X, Sureda A, Jafari S, et al. Berberine in Cardiovascular and Metabolic Diseases: From Mechanisms to Therapeutics. Theranostics. 2019;9(7):1923-51. doi:10.7150/thno.30787.

19. Yan L, Yan K, Kun W, et al. Berberine inhibits the migration and invasion of T24 bladder cancer cells via reducing the expression of heparanase. Tumor Biol. 2013;34(1):215-21. doi:10.1007/s13277012-0531-z.

20. Yan K, Zhang C, Feng J, et al. Induction of G1 cell cycle arrest and apoptosis by berberine in bladder cancer cells. Eur J Pharmacol. 2011;661(1-3):1-7. doi:10.1016/j.ejphar.2011.04.021.

21. Liu X, Cui J, Gong L, et al. The CUL4B-miR-372/373-PIK3CA-AKT axis regulates metastasis in bladder cancer. Oncogene. 2020;39(17):3588-603. doi:10.1038/s41388-020-1236-1.

22. Wang $Y$, Chen S, Sun S, et al. Wogonin Induces Apoptosis and Reverses Sunitinib Resistance of Renal Cell Carcinoma Cells via Inhibiting CDK4-RB Pathway. Front Pharmacol. 2020;11:1152. doi:10.3389/fphar.2020.01152.

23. Sun $S$, Zhang $X, X u M$, et al. Berberine downregulates CDC6 and inhibits proliferation via targeting JAK-STAT3 signaling in keratinocytes. Cell Death Dis. 2019;10(4):274. doi:10.1038/s41419-0191510-8.

24. Kim DE, Dollé MET, Vermeij WP, et al. Deficiency in the DNA repair protein ERCC1 triggers a link between senescence and apoptosis in human fibroblasts and mouse skin. Aging Cell. 2020;19(3):113. doi:10.1111/acel.13072.

25. Wang L, Li K, Lin X, et al. Metformin induces human esophageal carcinoma cell pyroptosis by targeting the miR-497/PELP1 axis. Cancer Lett. 2019;450:22-31. doi:10.1016/j.canlet.2019.02.014.

26. Johnson DE, O'Keefe RA, Grandis JR. Targeting the IL-6/JAK/STAT3 signalling axis in cancer. Nat Rev Clin Oncol. 2018;15(4):234-48. doi:10.1038/nrclinonc.2018.8.

27. Zhao D, Besser AH, Wander SA, et al. Cytoplasmic p27 promotes epithelial-mesenchymal transition and tumor metastasis via STAT3-mediated Twist1 upregulation. Oncogene. 2015;34(43):5447-59. doi:10.1038/onc.2014.473. 
28. Yang Z, He L, Lin K, et al. The KMT1A-GATA3-STAT3 Circuit Is a Novel Self-Renewal Signaling of Human Bladder Cancer Stem Cells. Clin Cancer Res. 2017;23(21):6673. doi:10.1158/1078-0432.CCR17-0882. LP - 6685.

29. Pan Q, Yang G-L, Yang J-H, et al. Metformin can block precancerous progression to invasive tumors of bladder through inhibiting STAT3-mediated signaling pathways. J Exp Clin cancer Res. 2015;34(1):77. doi:10.1186/s13046-015-0183-0.

30. Tsang CM, Cheung YC, Lui VWY, et al. Berberine suppresses tumorigenicity and growth of nasopharyngeal carcinoma cells by inhibiting STAT3 activation induced by tumor associated fibroblasts. BMC Cancer. 2013;13:619. doi:10.1186/1471-2407-13-619.

31. Yu Y-N, Yu T-C, Zhao H-J, et al. Berberine may rescue Fusobacterium nucleatum-induced colorectal tumorigenesis by modulating the tumor microenvironment. Oncotarget. 2015;6(31):32013-26. doi:10.18632/oncotarget.5166.

32. Liu X, Ji Q, Ye N, et al. Berberine Inhibits Invasion and Metastasis of Colorectal Cancer Cells via COX2/PGE2 Mediated JAK2/STAT3 Signaling Pathway. PLoS One. 2015;10(5):e0123478-8. doi:10.1371/journal.pone.0123478.

33. Wang J, Yang S, Cai X, et al. Berberine inhibits EGFR signaling and enhances the antitumor effects of EGFR inhibitors in gastric cancer. Oncotarget. 2016;7(46):76076-86. doi:10.18632/oncotarget.12589.

34. Yang $Y$, Wang Q, Xie M, et al. Berberine exerts an anti-inflammatory role in ocular Behcet's disease. Mol Med Rep. 2017;15(1):97-102. doi:10.3892/mmr.2016.5980.

35. Liu L, Luo N, Guo J, Xie Y, Chen L, Cheng Z. Berberine inhibits growth and inflammatory invasive phenotypes of ectopic stromal cells: Imply the possible treatment of adenomyosis. J Pharmacol Sci. 2018;137(1):5-11. doi:10.1016/j.jphs.2017.12.001.

36. Sha W, Shen L, Zhou L, Xu D, Yang J, Lu G. Silencing of CXCL12 performs a protective effect on C5b9-induced injury in podocytes. Int Urol Nephrol. 2018;50(8):1535-44. doi:10.1007/s11255-018-17998.

37. Zhu L, Gu PQ, Shen H. Protective effects of berberine hydrochloride on DSS-induced ulcerative colitis in rats. Int Immunopharmacol. 2019;68:242-51. doi:10.1016/j.intimp.2018.12.036.

38. Liu X, Zhang X, Ye L, Yuan H. Protective mechanisms of berberine against experimental autoimmune myocarditis in a rat model. Biomed Pharmacother. 2016;79:222-30.

doi:10.1016/j.biopha.2016.02.015.

39. O'Shea JJ, Schwartz DM, Villarino AV, Gadina M, McInnes IB, Laurence A. The JAK-STAT pathway: Impact on human disease and therapeutic intervention. Annu Rev Med. 2015;66:311-28. doi:10.1146/annurev-med-051113-024537.

40. Mascarenhas JO, Rampal RK, Kosiorek HE, et al. Phase 2 study of ruxolitinib and decitabine in patients with myeloproliferative neoplasm in accelerated and blast phase. Blood Adv. 2020;4(20):5246-56. doi:10.1182/bloodadvances.2020002119. 
41. Fendler A, Stephan C, Yousef GM, Jung K. MicroRNAs as Regulators of Signal Transduction in Urological Tumors. Clin Chem. 2011;57(7):954-68. doi:10.1373/clinchem.2010.157727.

42. Servais FA, Kirchmeyer M, Hamdorf M, et al. Modulation of the IL-6-Signaling Pathway in Liver Cells by miRNAs Targeting gp130, JAK1, and/or STAT3. Mol Ther Nucleic Acids. 2019;16:419-33. doi:10.1016/j.omtn.2019.03.007.

43. de Pontual L, Yao E, Callier P, et al. Germline deletion of the miR-17 92 cluster causes skeletal and growth defects in humans. Nat Genet. 2011;43(10):1026-30. doi:10.1038/ng.915.

44. Ventura A, Young AG, Winslow MM, et al. Targeted deletion reveals essential and overlapping functions of the miR-17 through 92 family of miRNA clusters. Cell. 2008;132(5):875-86. doi:10.1016/j.cell.2008.02.019.

45. Jiang S, Li C, Olive V, et al. Molecular dissection of the miR-17-92 cluster's critical dual roles in promoting Th1 responses and preventing inducible Treg differentiation. Blood. 2011;118(20):548797. doi:10.1182/blood-2011-05-355644.

46. Gu J, Wang D, Zhang J, et al. GFRa2 prompts cell growth and chemoresistance through downregulating tumor suppressor gene PTEN via Mir-17-5p in pancreatic cancer. Cancer Lett. 2016;380(2):434-41. doi:10.1016/j.canlet.2016.06.016.

47. Yang $X$, Du WW, Li H, et al. Both mature miR-17-5p and passenger strand miR-17-3p target TIMP3 and induce prostate tumor growth and invasion. Nucleic Acids Res. 2013;41(21):9688-704. doi:10.1093/nar/gkt680.

48. Zeng A, Yin J, Wang Z, et al. miR-17-5p-CXCL14 axis related transcriptome profile and clinical outcome in diffuse gliomas. Oncoimmunology. 2018;7(12):e1510277. doi:10.1080/2162402X.2018.1510277.

49. Wei Q, Li Y-X, Liu M, Li X, Tang H. MiR-17-5p targets TP53INP1 and regulates cell proliferation and apoptosis of cervical cancer cells. IUBMB Life. 2012;64(8):697-704. doi:10.1002/iub.1051.

50. Cheng YY, Wright CM, Kirschner MB, et al. KCa1.1, a calcium-activated potassium channel subunit alpha 1 , is targeted by miR-17-5p and modulates cell migration in malignant pleural mesothelioma. Mol Cancer. 2016;15(1):44. doi:10.1186/s12943-016-0529-z.

51. Liu D-L, Lu L-L, Dong L-L, et al. miR-17-5p and miR-20a-5p suppress postoperative metastasis of hepatocellular carcinoma via blocking HGF/ERBB3-NF-KB positive feedback loop. Theranostics. 2020;10(8):3668-83. doi:10.7150/thno.41365.

52. Li J, Lai Y, Ma J, et al. miR-17-5p suppresses cell proliferation and invasion by targeting ETV1 in triple-negative breast cancer. BMC Cancer. 2017;17(1):745. doi:10.1186/s12885-017-3674-x.

53. Hossain A, Kuo MT, Saunders GF. Mir-17-5p regulates breast cancer cell proliferation by inhibiting translation of AlB1 mRNA. Mol Cell Biol. 2006;26(21):8191-201. doi:10.1128/MCB.00242-06.

54. Zhang M, Liu Q, Mi S, et al. Both miR-17-5p and miR-20a alleviate suppressive potential of myeloidderived suppressor cells by modulating STAT3 expression. J Immunol. 2011;186(8):4716-24. doi:10.4049/jimmunol.1002989. 
55. Yang ZG, Awan FM, Du WW, et al. The Circular RNA Interacts with STAT3, Increasing Its Nuclear Translocation and Wound Repair by Modulating Dnmt3a and miR-17 Function. Mol Ther. 2017;25(9):2062-74. doi:10.1016/j.ymthe.2017.05.022.

56. Shen Y, Tong Z-W, Zhou Y, et al. Inhibition of IncRNA-PAX8-AS1-N directly associated with VEGF/TGFB1/8-OhdG enhances podocyte apoptosis in diabetic nephropathy. Eur Rev Med Pharmacol Sci. 2020;24(12):6864-72. doi:10.26355/eurrev_202006_21676.

57. Du W, Pan Z, Chen X, et al. By Targeting Stat3 microRNA-17-5p Promotes Cardiomyocyte Apoptosis in Response to Ischemia Followed by Reperfusion. Cell Physiol Biochem. 2014;34(3):955-65. doi:10.1159/000366312.

58. Wang Z, Yuan W, Li B, et al. PEITC promotes neurite growth in primary sensory neurons via the miR17-5p/STAT3/GAP-43 axis. J Drug Target. 2019;27(1):82-93. doi:10.1080/1061186X.2018.1486405.

59. Najm A, Masson F-M, Preuss $P$, et al. miR-17-5p reduces inflammation and bone erosions in collagen induced arthritis mice and directly targets the JAK-STAT pathway in rheumatoid arthritis fibroblastlike synoviocytes. Arthritis Rheumatol. 2020;72(12):2030-9. doi:10.1002/art.41441.

60. Diogo CV, Machado NG, Barbosa IA, Serafim TL, Burgeiro A, Oliveira PJ. Berberine as a promising safe anti-cancer agent - is there a role for mitochondria? Curr Drug Targets. 2011;12(6):850-9. doi:10.2174/138945011795528930.

\section{Figures}


a

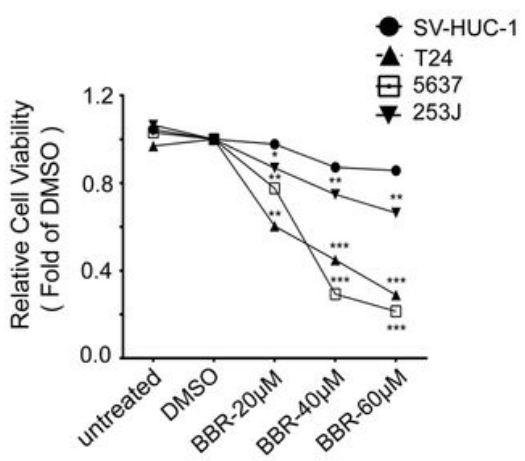

b

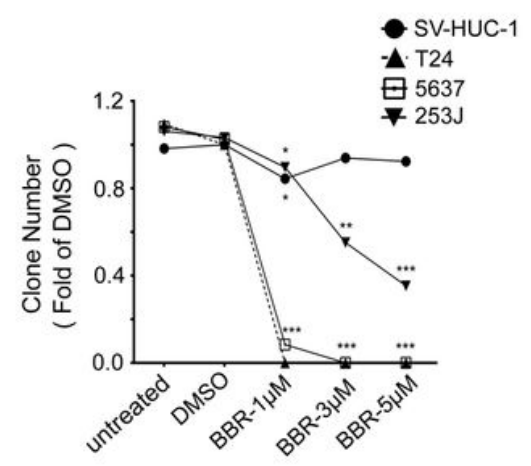

C

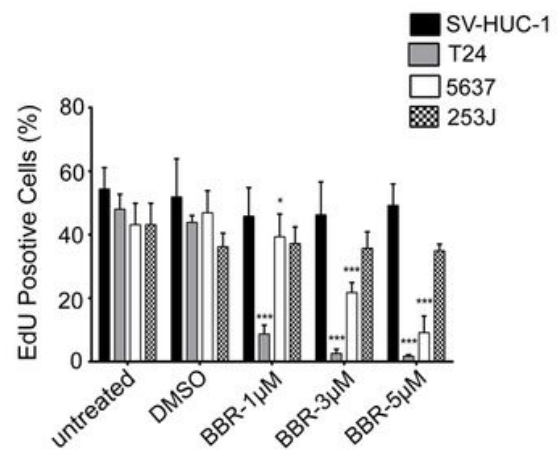

e d

T24

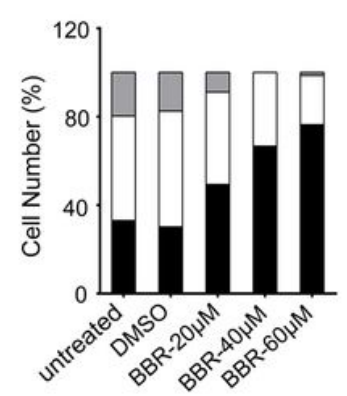

f
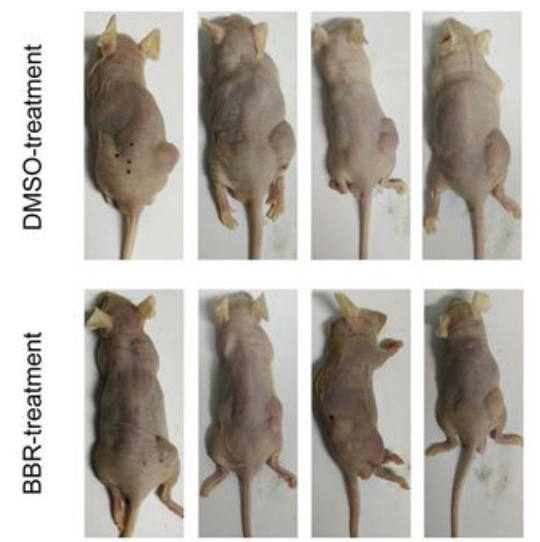

5637

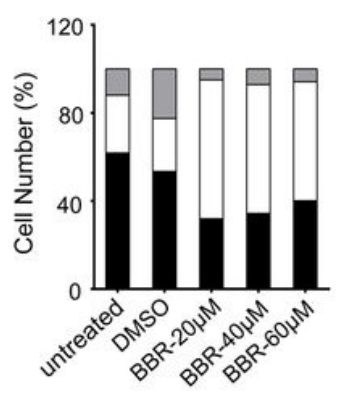

g

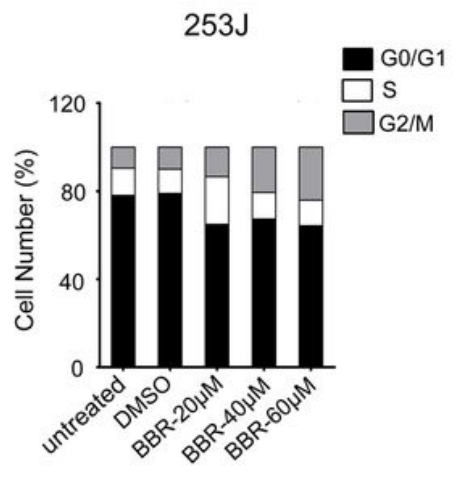

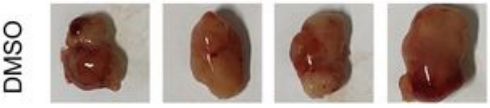

$\underset{\substack{\alpha \\ \hdashline}}{\stackrel{\alpha}{\infty}}$
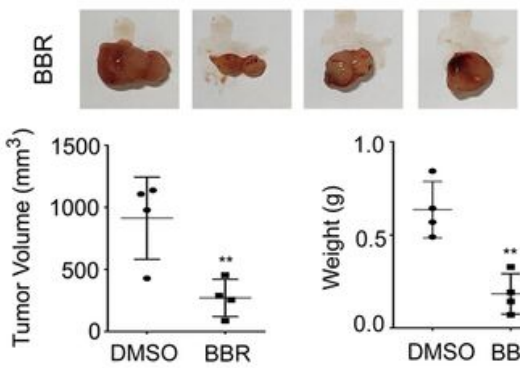

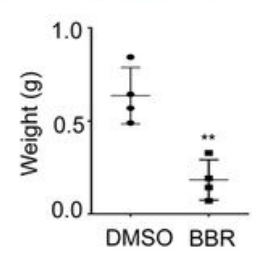

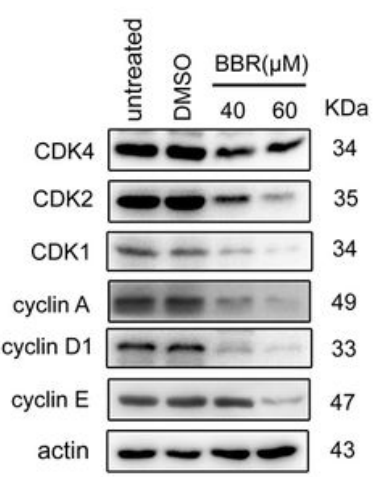

$\mathrm{h}$
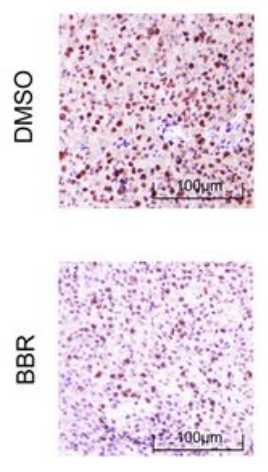

Figure 1

BBR inhibits proliferation of BCa cells both in vitro and vivo. (a) T24, 5637, 253 J and SV-HUC-1 cells were treated with different concentrations of BBR for 48 hours, and cell viabilities were assessed using MTT assays. (b) Colony formation assays of T24, 5637, 253J and SV-HUC-1 cells treated with BBR at different concentrations. (c) EdU incorporation assays of T24, 5637, 253 J and SV-HUC-1 cells treated with different concentrations of BBR for 48 hours. (d) The cell cycle distribution was analyzed by flow cytometry in T24, 5637 and 253 J cells treated with different concentrations of BBR for 48 hours. (e) T24 cells were treated with BBR at different concentrations for 48 hours and protein expression levels of indicated proteins were determined by western blot. (f-g) Tumor formation by T24 cells in nude mice. 
$5 \times 106$ T24 cells were subcutaneously injected into nude mice. Mice were treated with BBR or DMSO once a day for 2 weeks. Then the mice were sacrificed, and nude-mice ( $f$ ) and xenografts ( $g$, upper) were photographed. The tumor volume and tumor weight ( $\mathrm{g}$, lower) were measured. (h) Representative images of Ki-67 expression assessed by IHC in xenografts from both the BBR-treatment group (lower冈and the DMSO-treatment group (upper). Scale bars, $100 \mu \mathrm{m}$. ${ }^{*} \mathrm{P}<0.05 ;{ }^{* \star \mathrm{P}}<0.01$; ${ }^{* \star *} \mathrm{P}<0.001$, compared with that of DMSO-treated cells.

a

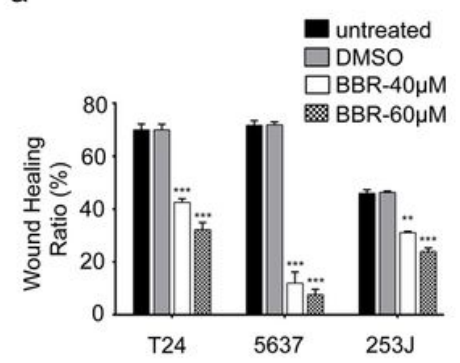

d

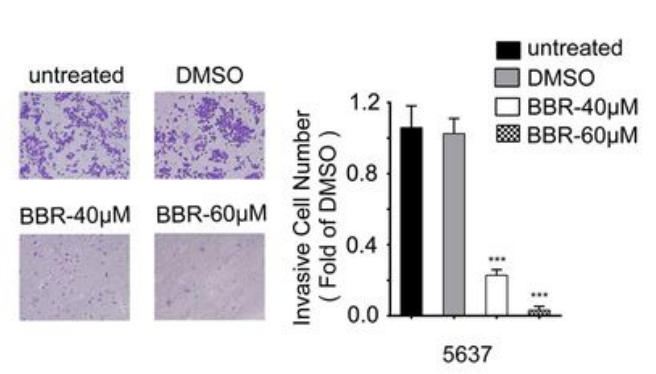

g

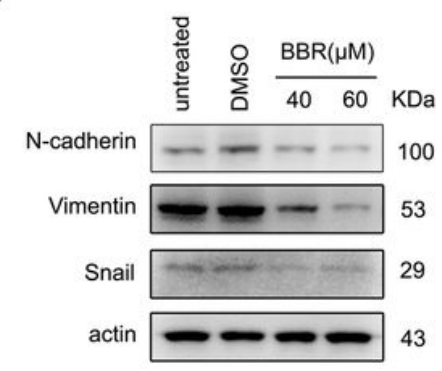

b

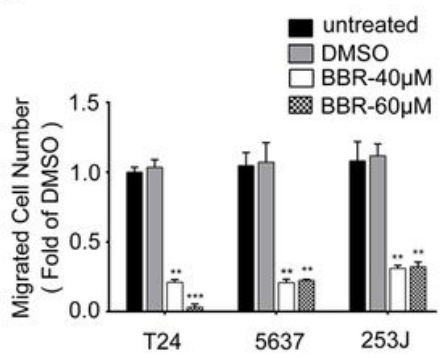

e

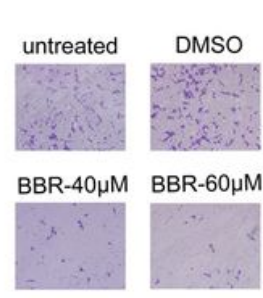

$\mathrm{h}$

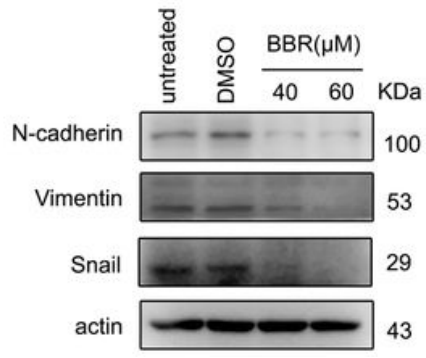

C

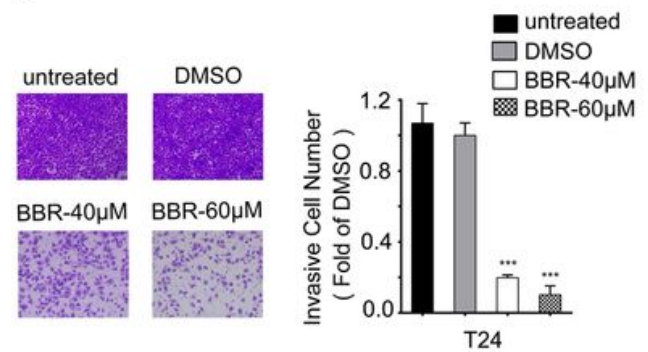

f

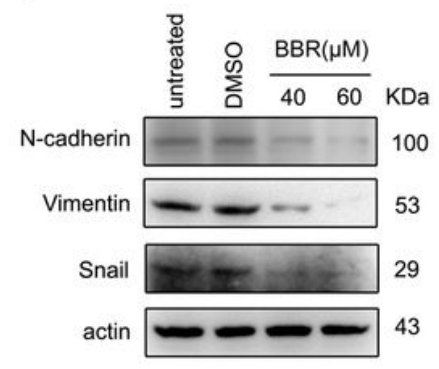

i

Figure 2

BBR represses migration and invasion of BCa cells. (a-e) T24, 5637 and 253J cells were treated with different concentrations of BBR, and cell migration and invasion abilities of BCa cells were measured by wound-healing assays (a), transwell migration assays (b) and transwell invasion assays (c-e). (f-h) T24 (f), $5637(\mathrm{~g})$ and $253 \mathrm{~J}(\mathrm{~h})$ cells were treated with BBR at different concentrations for 48 hours and protein expression levels of indicated proteins were determined by western blot. (i) T24 cells were treated with BBR at different concentrations for 48 hours. The mRNA expression levels of MMP2 and MMP9 were detected by qRT-PCR analysis. The normalized expression levels in cells treated with DMSO was set as 1 . $\star \star P<0.01 ; * \star * P<0.001$, compared with that of DMSO-treated cells. 
a

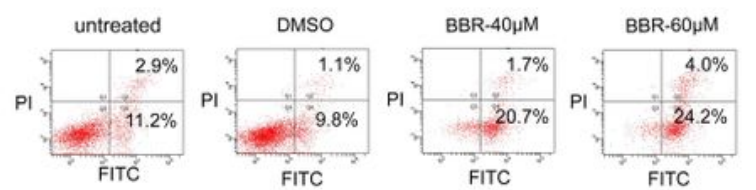

C

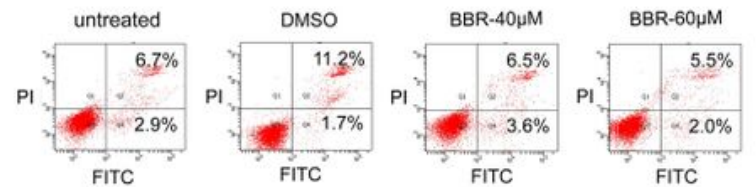

e

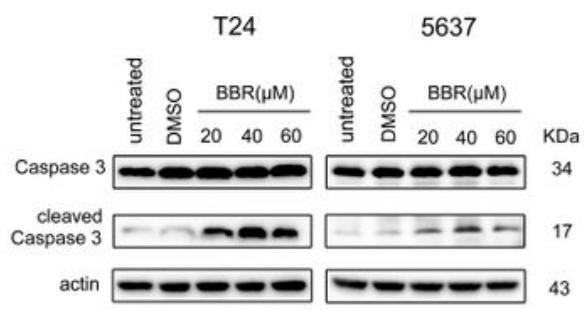

$\mathrm{h}$

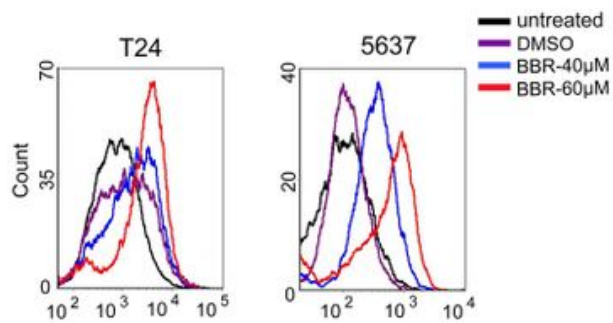

k
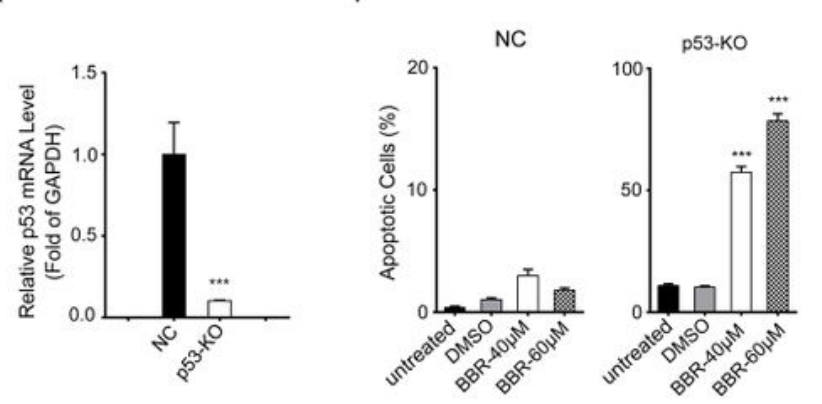

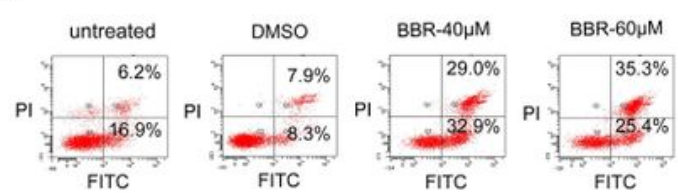

d

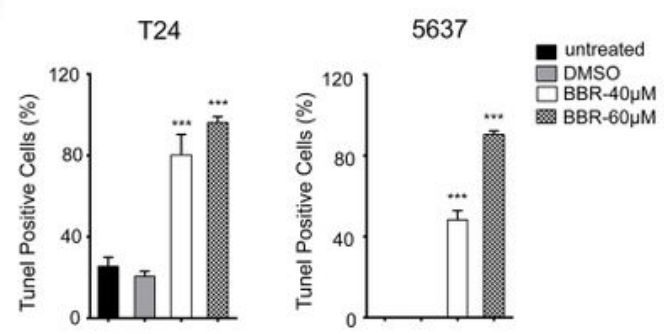

f

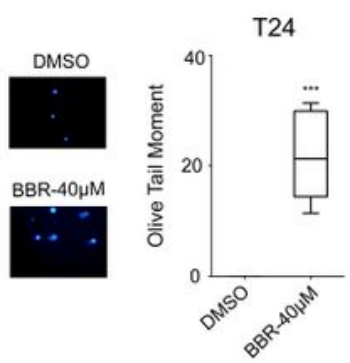

g

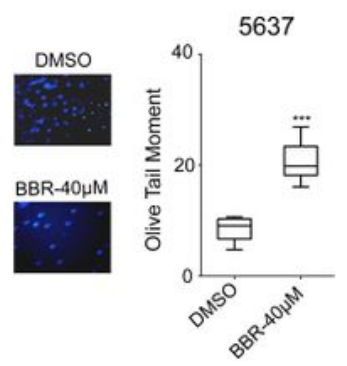

j

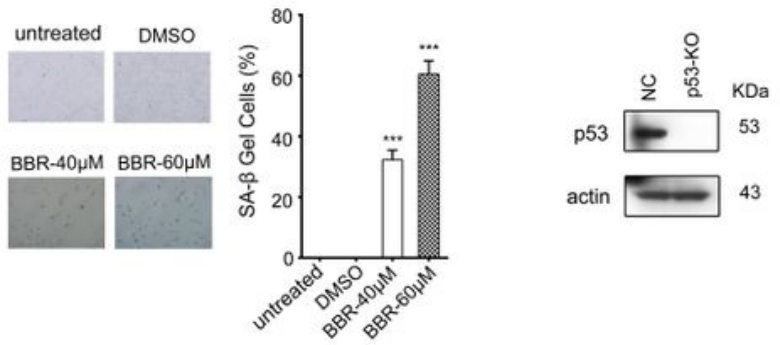

m
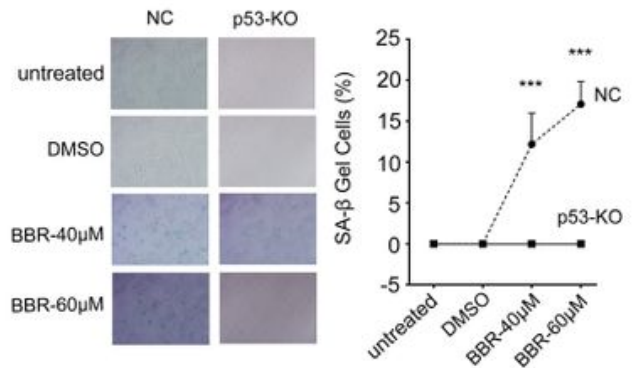

Figure 3

BBR induces apoptosis or senescence in BCa cells in a p53-dependent way. (a-c) Flow cytometric analysis of Annexin V-FITC/PI-stained T24 (a), 5637 (b) and 253J (c) cells treated with different concentrations of BBR for 72 hours. (d) Tunel assays of T24 and 5637 cells treated with different concentrations of BBR for 72 hours. (e) T24 and 5637 cells were treated with different concentrations of BBR for 72 hours. Caspase 3 and cleaved-caspase 3 expression levels were determined by western blot. 


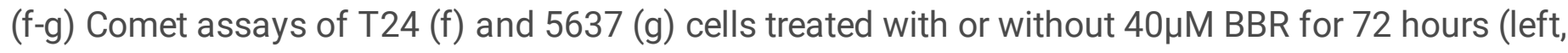
representative images of Comet assay, right, box plots indicating the Olive tail moment). (h) T24 and 5637 cells were treated with or without BBR for 72 hours and stained with DCFH-DA probe. ROS levels were determined by flow cytometry. (i) SA- $\beta-G a l$ staining of $253 \mathrm{~J}$ cells treated with different concentrations of BBR (left, representative images of SA- $\beta$-Gal staining, right, bar chart indicating the average SA- $\beta-G$ al cells percentage). ( $j-k)$ The protein $(j)$ and mRNA ( $k$ ) expression levels of $p 53$ in indicated $253 \mathrm{~J}$ cells were determined by western blot and qRT-PCR. The normalized mRNA expression in negative control (NC) cells was set as 1. (I-m) The indicated 253J cells were treated with different concentrations of BBR for 72 hours. The apoptosis was measured by flow cytometry (I), and senescence was determined by SA- $\beta$-Gal staining $(\mathrm{m}) .{ }^{\star \star *} \mathrm{P}<0.001$, compared with that of DMSO-treated cells. 


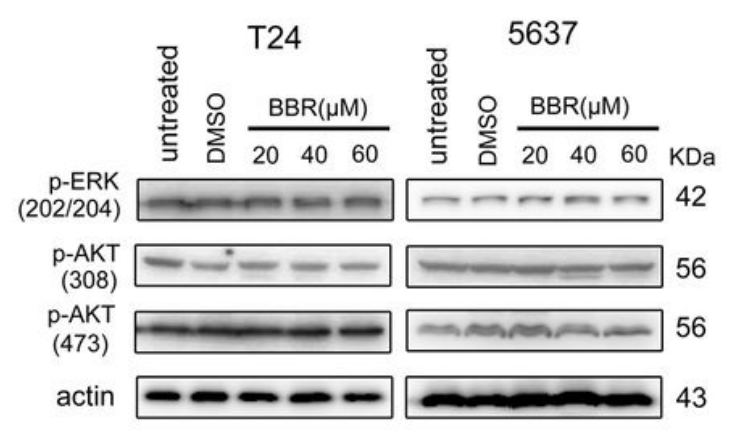

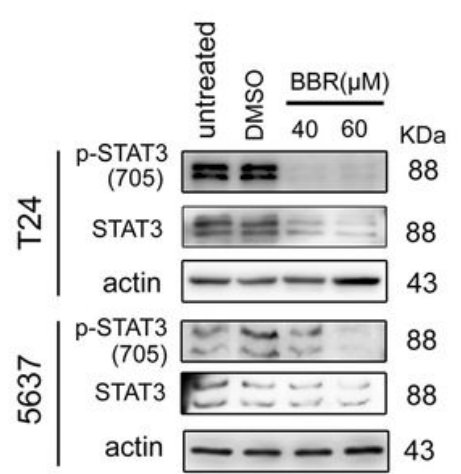

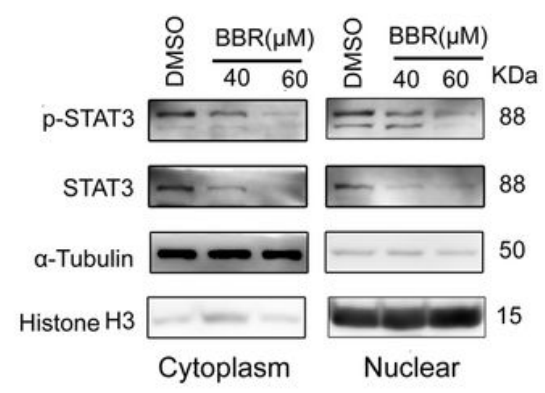

f

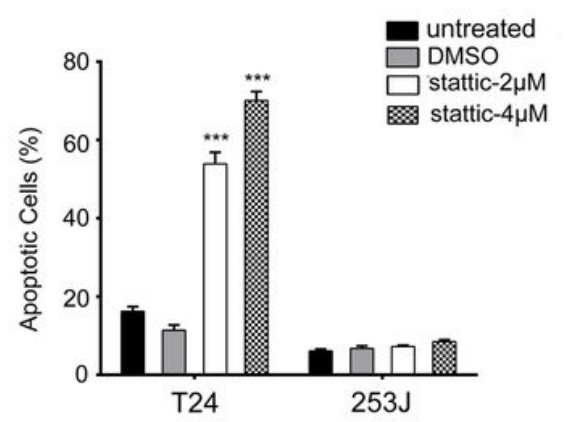

i

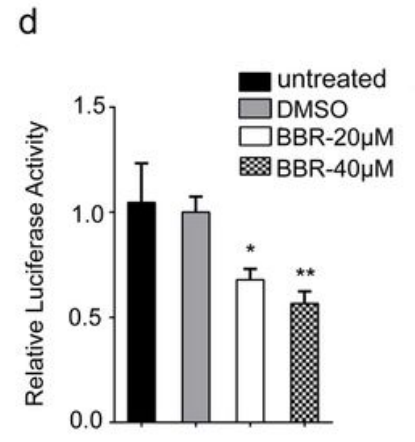

g
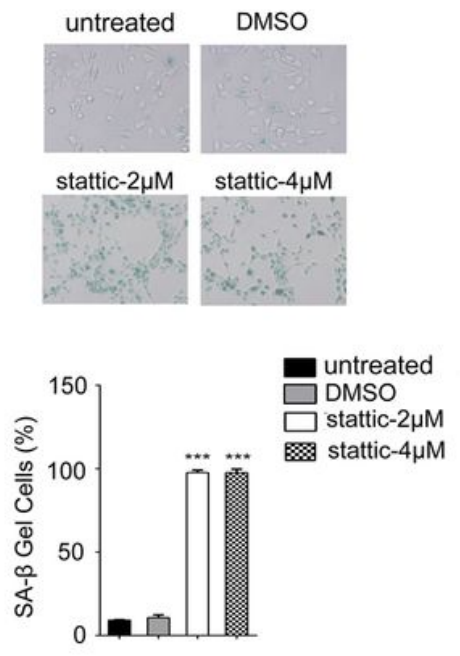

e

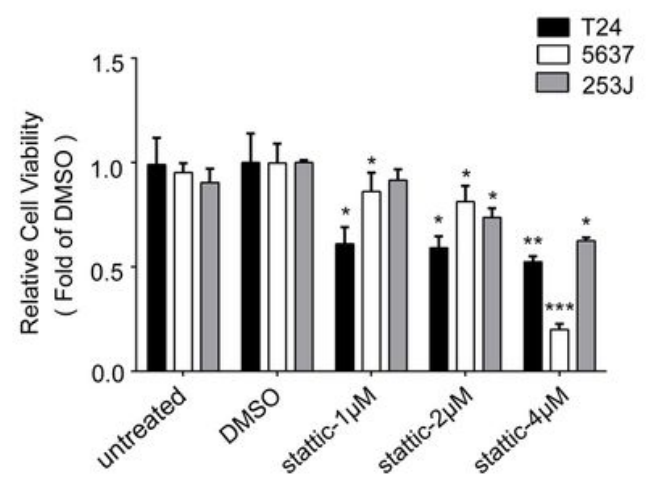

h
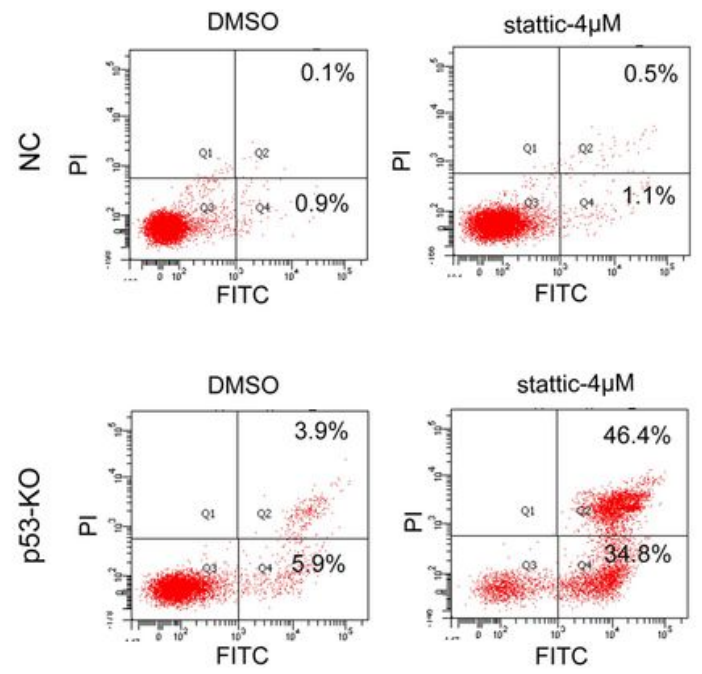
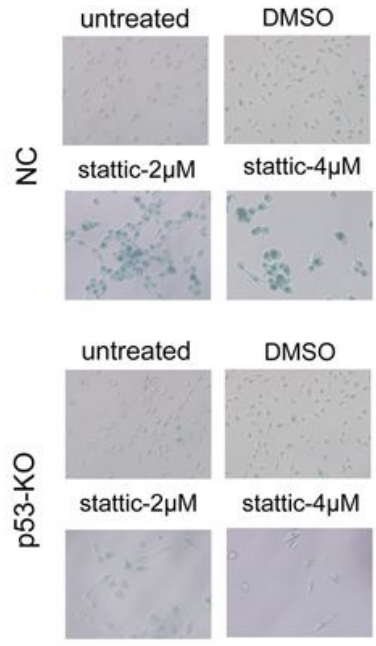

Figure 4

BBR down-regulates expression and activity of STAT3 in BCa cells. (a) T24 and 5637 cells were treated with different concentrations of BBR for 48 hours, and expression levels of indicated proteins were determined by western blot. (b) Western blot analysis of STAT3 and p-STAT3 levels in T24 and 5637 cells after treatment with different concentrations of BBR for 48 hours. (c) 5637 cells were treated with different concentrations of BBR for 48 hours, and cytoplasm and nuclear fractions were extracted to determine protein expression levels of STAT3 and p-STAT3 by western blot. (d) T24 cells were transfected 
with STAT3 responsive luciferase reporters. After 24 hours, cells were treated with different concentrations of BBR for another 24 hours and luciferase assays were performed. The normalized luciferase activity of DMSO-treated cells was set as 1 . (e) T24, 5637 and 253J cells were treated with different concentrations of stattic for 48 hours, and cell viabilities were assessed using MTT assays. (f) T24 and 253J cells treated with different concentrations of stattic for 72 hours and cell apoptosis was measured by flow cytometry. (g) SA- $\beta$-Gal staining of $253 \mathrm{~J}$ cells treated with different concentrations of stattic (upper, representative images of SA- $\beta$-Gal staining, lower, bar charts indicating the average SA- $\beta$ Gal cells percentage). (h) Flow cytometric analysis of apoptosis of NC (upper) and p53-KO (lower) 253J cells treated with different concentrations of stattic for 72 hours. (i) The NC and p53-KO 253J cells were treated with different concentrations of stattic, and senescence was determined by SA- $\beta$-Gal staining. *, $P$ $<0.05 ; \star \star, P<0.01 * \star \star P<0.001$ compared to that of DMSO-treated cells. 
a

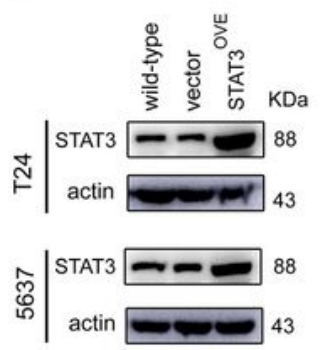

d

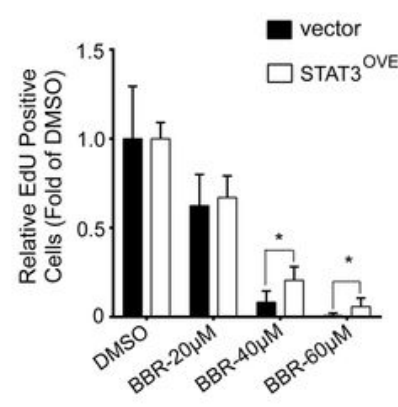

h

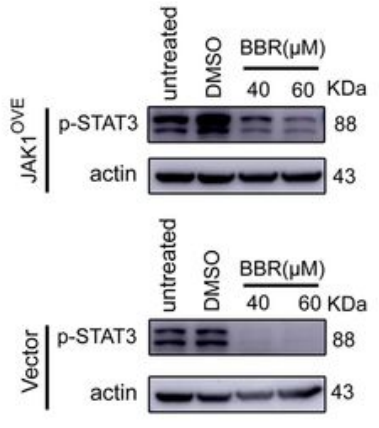

I

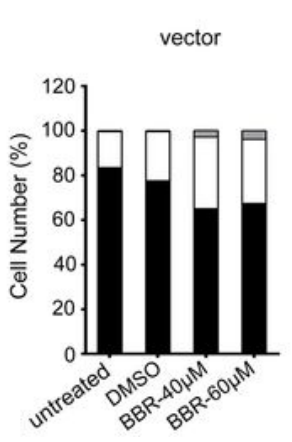

b

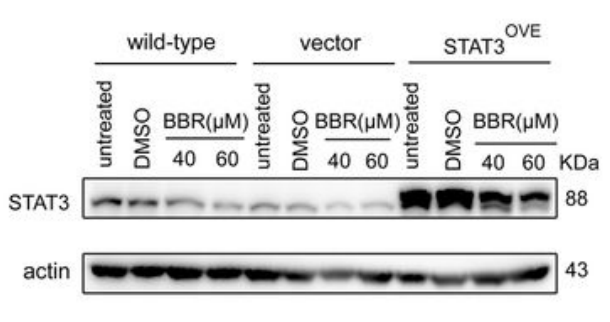

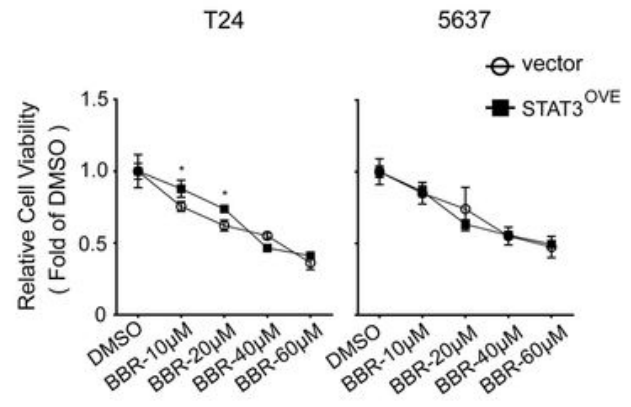

e

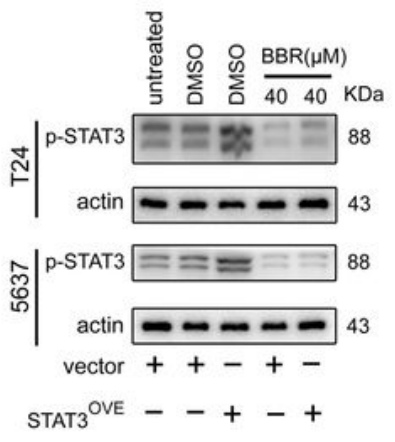

i

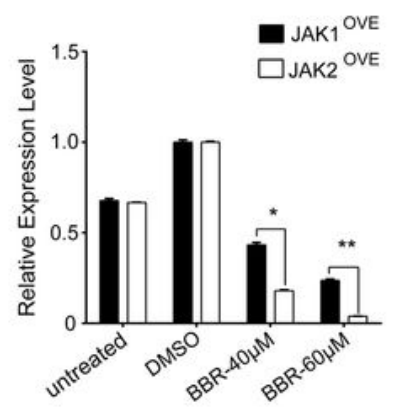

f

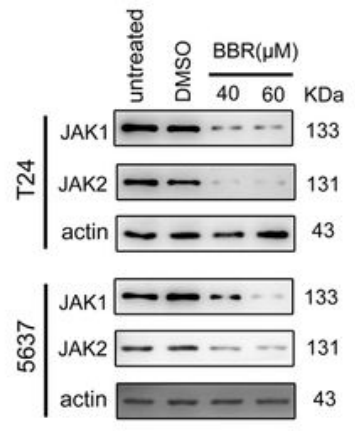

j
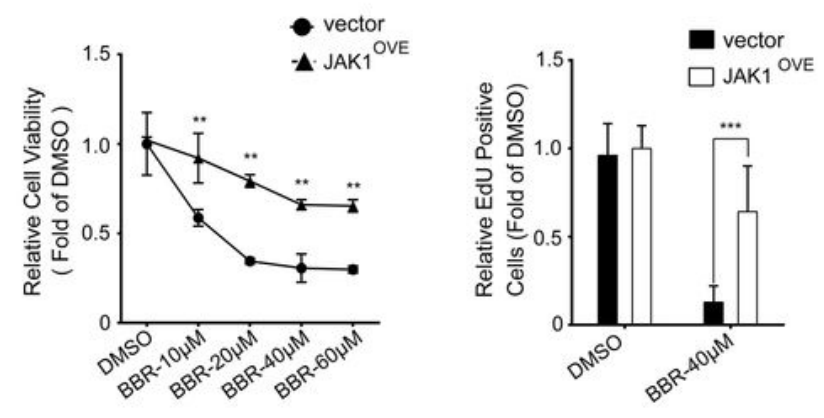

m

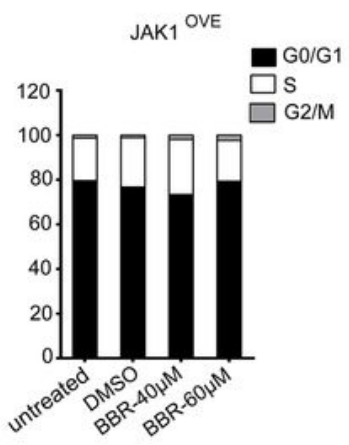

g

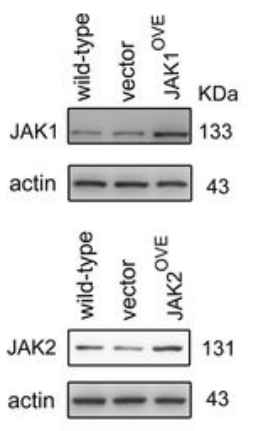

k
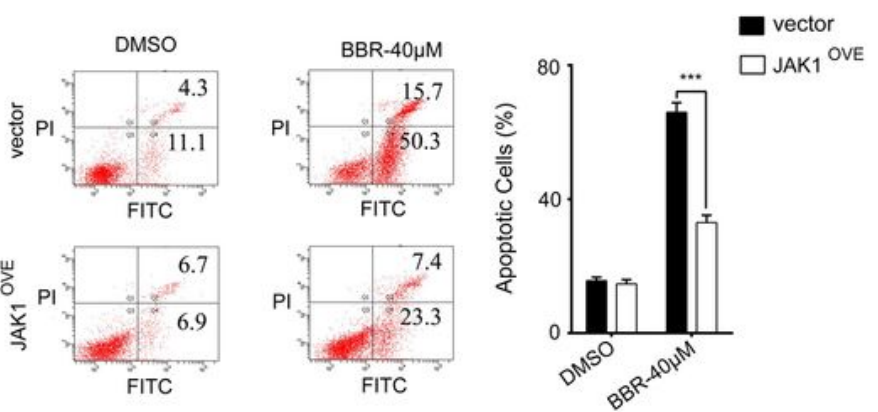

\section{Figure 5}

BBR down-regulates expression of JAK1 to inhibit activity of STAT3. (a) Protein expression levels of STAT3 in indicated T24 (upper) and 5637 (lower) cells were determined by western blot. (b) Western blot analysis of total STAT3 levels in indicated 5637 cells with BBR treatment. (c) Cell viabilities of indicated T24 (left) and 5637 (right) cells treated with BBR were determined by MTT assay. (d) DNA replication of indicated 5637 cells treated with BBR was determined by EdU incorporation assays. *, P < 0.05 , compared 
to that of cells stably transfected with empty vector under the same treatment. (e) Western blot analysis of p-STAT3 levels in indicated T24 and 5637 cells treated with BBR for 48 hours. (f) Western blot analysis of JAK1 and JAK2 levels in T24 and 5637 cells with BBR treatment for 48 hours. (g) Western blot analysis of JAK1 and JAK2 levels in indicated 5637 cells. (h-i) Western blot analysis (h) of p-STAT3 levels in indicated 5637 cells after treatment with different concentrations of BBR for 48 hours. (i) Quantitative analysis of (h). Band intensity was quantitated by densitometric scanning and relative expression level was normalized to actin. The normalized relative expression level of DMSO-treated cells was set as $1 .{ }^{*}, \mathrm{P}$ $<0.05$; $\star \star, ~ P<0.01$ compared to that of cells stably transfected with vector expressing JAK2 under same treatment. (j-m) Cell viability and DNA replication of indicated 5637 cells treated BBR were determined by MTT assay (j) and EdU incorporation assays ( $k$ ). Cell cycle distribution $(\mathrm{l})$ and apoptosis $(\mathrm{m})$ of that were determined by flow cytometry. ${ }^{*}, \mathrm{P}<0.05,{ }^{\star} *, \mathrm{P}<0.01$; $* \star * \mathrm{P}<0.001$ compared to that of cells stably transfected with empty vector under same treatment.

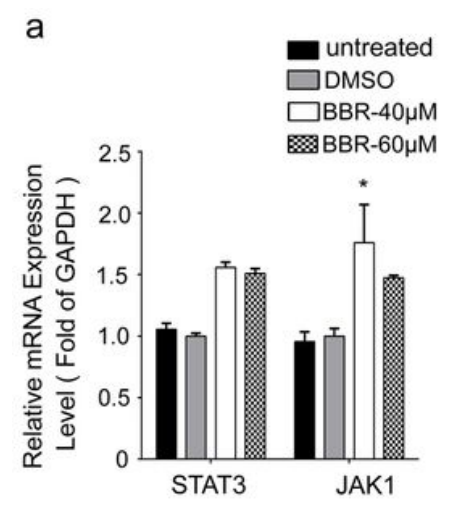

d

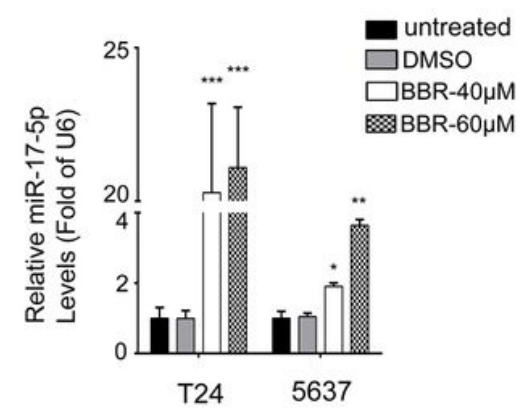

g

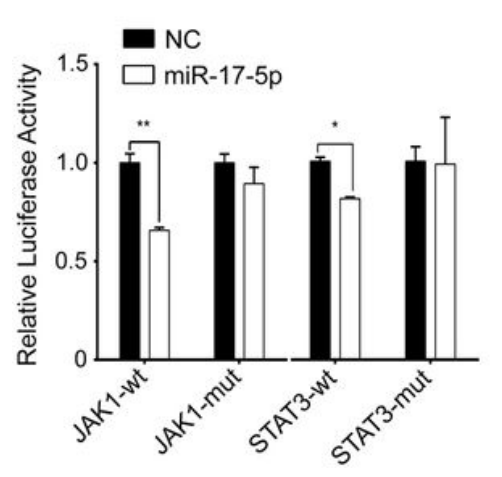

b

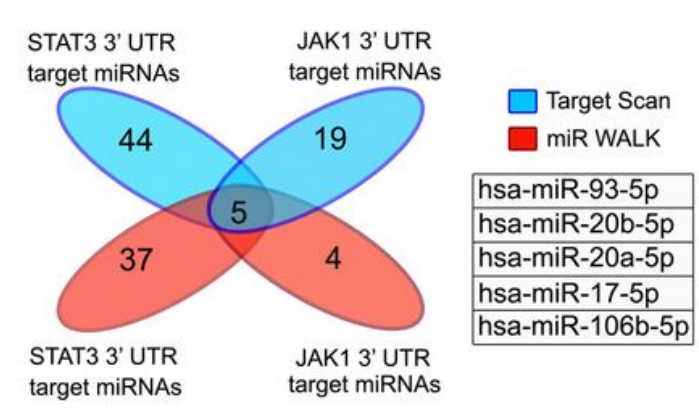

e

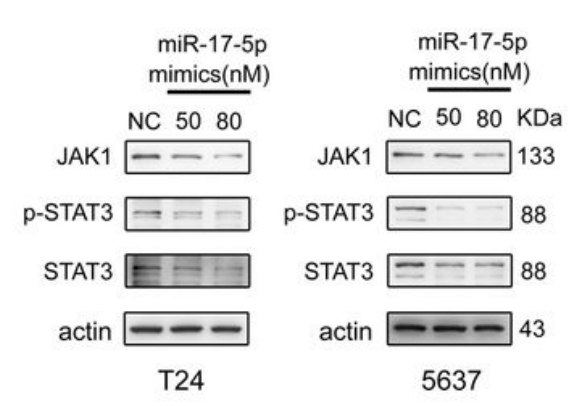

$\mathrm{h}$

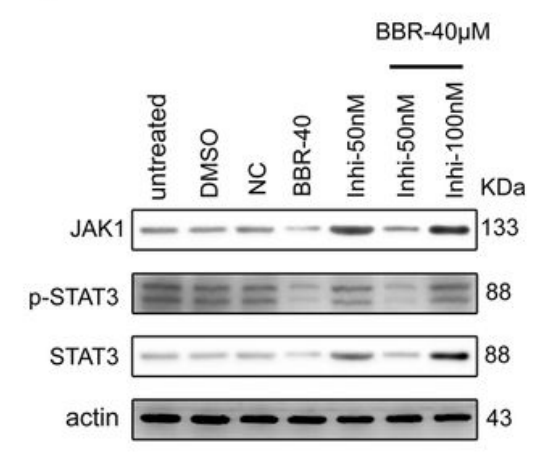

$\mathrm{C}$

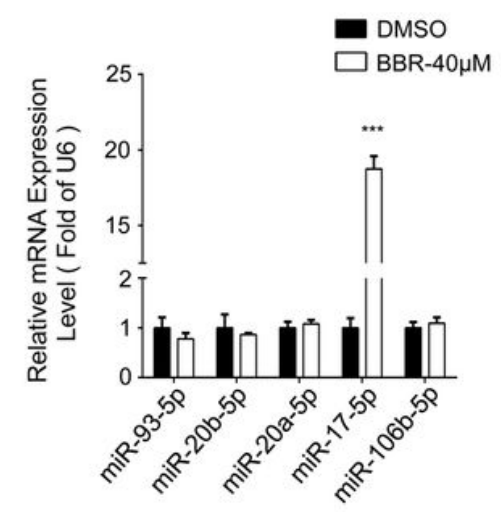

f

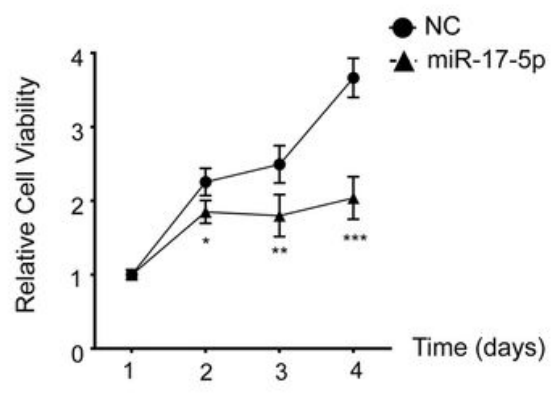

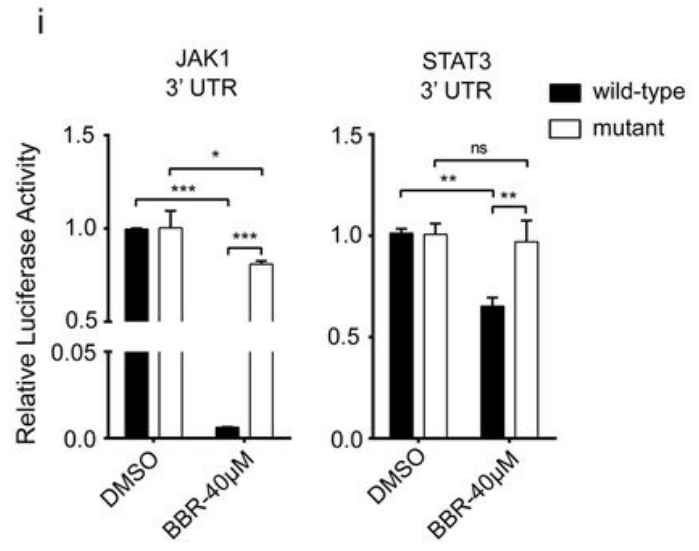




\section{Figure 6}

BBR represses expression of JAK1 and STAT3 by up-regulating miR-17-5p in vitro. (a) The mRNA expression levels of JAK1 and STAT3 in 5637 cells treated with BBR for 48 hours were detected by qRTPCR analysis. (b) Venn diagram showed potential miRNAs targeting JAK1 or STAT3 predicted by TargetScan or miR WALK. (c) The indicated miRNAs expression levels in 5637 cells treated with or without BBR for 48 hours were determined by qRT-PCR analysis. (d) qRT-PCR analysis of miR-17-5p expression levels in cells with BBR treatment for 48 hours. Normalized expression levels in cells treated with DMSO was set as $1 .{ }^{\star}, \mathrm{P}<0.05$; $* \star, P<0.01 * \star \star \mathrm{P}<0.001$ compared with that of DMSO-treated cells. (e) The levels of indicated proteins were assessed by western blot in T24 (left) and 5637 (right) cells transfected with miR-17-5p mimics or NC. (f) 5637 cells were transfected with miR-17-5p mimics or NC, and cell proliferation was assessed using MTT assays. Normalized viability of each indicated cell line on day1 was set as $1 . *, \mathrm{P}<0.05 ; * \star, \mathrm{P}<0.01 * \star \star \mathrm{P}<0.001$ compared to that of cells transfected with NC. (g) Luciferase assays were performed in T24 cells transfected with indicated 3'UTR constructs and miR-17$5 p$ mimics or NC. Normalized luciferase activity of cells transfected with $\mathrm{NC}$ was set as $1 .{ }^{*} \mathrm{P}<0.05$; ${ }^{* *} \mathrm{P}<$ 0.01. (h) 5637 cells were transfected with miR-17-5p inhibitors or NC for 6 hours followed by treatment with or without $40 \mu \mathrm{M}$ BBR. The indicated protein levels were determined by western blot. (i) T24 cells were transfected with indicated $3^{\prime} U T R$ constructs. After 24 hours, cells were treated with or without $40 \mu M$ BBR for another 24 hours and luciferase assays were performed. Normalized luciferase activity of cells treated with DMSO was set as 1 . *, $\mathrm{P}<0.05$; **, $\mathrm{P}<0.01$ *** $\mathrm{P}<0.001$; ns no significant.

a

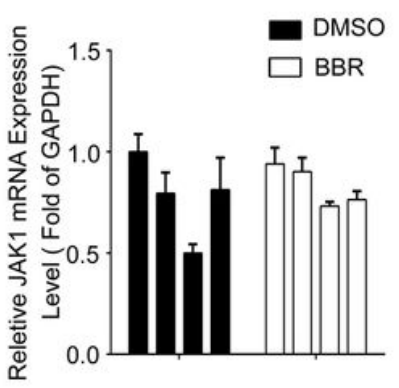

b

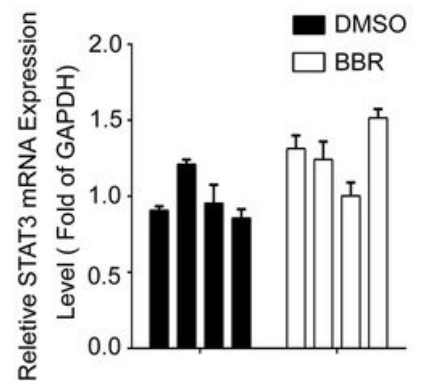

C

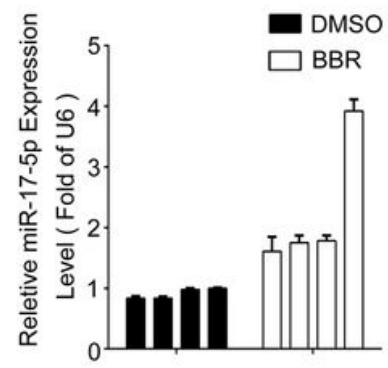

d

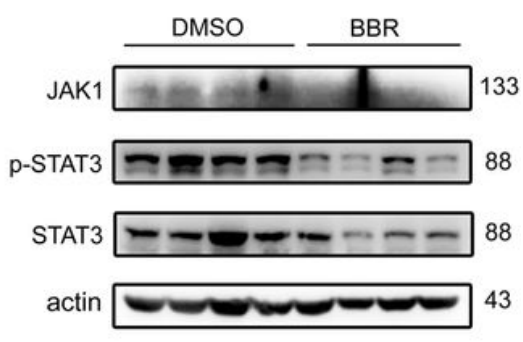

e

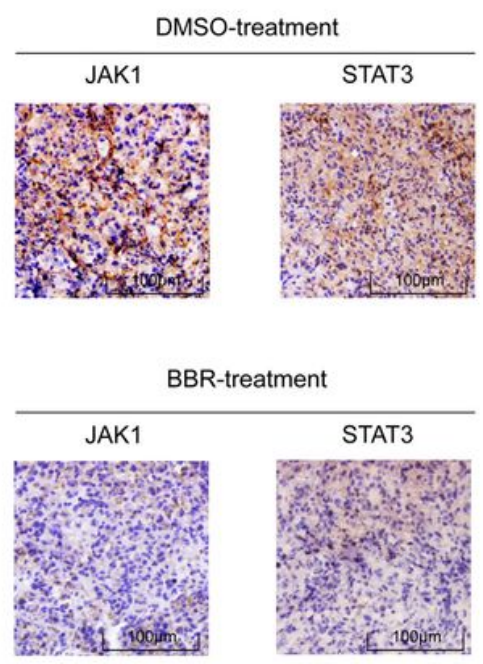

f

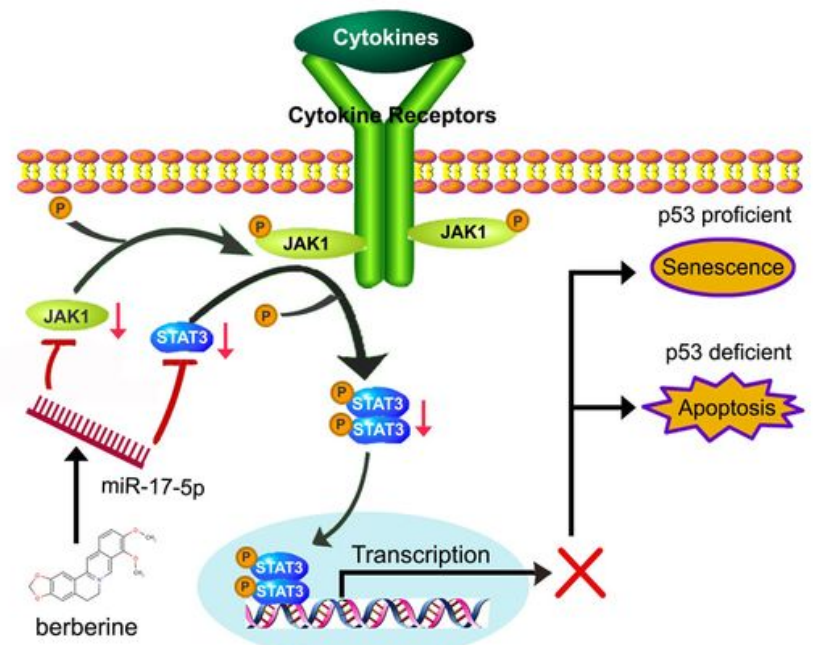




\section{Figure 7}

The effect of BBR on miR-17-5p-JAK1-STAT3 pathway in vivo. (a-c) qRT-PCR analysis on xenografted tumors from BBR-treatment group and DMSO-treatment group to assess expression levels of JAK1 (a), STAT3 (b) and miR-17-5p (c). Each column in every bar chart represented relative mRNA or miRNA expression level of one sample from indicated treatment group. (d) Western blot was performed on xenografted tumors from BBR-treatment group and DMSO-treatment group to measure protein expression levels of JAK1, p-STAT3 and STAT3. (e) IHC staining of JAK1 (left) and STAT3 (right) in xenografted tumors from both the BBR-treatment group and the DMSO-treatment group. Scale bars, $100 \mu \mathrm{m}$. (f) Schematic model of underlying molecular mechanism of BBR to exerts anti-tumor effects in BCa. $P$, phosphate.

\section{Supplementary Files}

This is a list of supplementary files associated with this preprint. Click to download.

- SupplementaryFigure1.jpg

- SupplementaryFigure2.jpg

- SupplementaryFigure3.jpg

- SupplementaryFigure4.jpg

- SupplementaryFigure5.jpg

- SupplementaryFigure6.jpg

- SupplementaryTable1.xlsx

- SupplementaryTable2.xlsx 